TRANSACTIONS OF THE

AMERICAN MATHEMATICAL SOCIETY

Volume 00, Number 0, Xxxx XXXX, Pages 3611-3643

S 0002-9947(97)01989-2

\title{
ARITHMETICITY, DISCRETENESS AND VOLUME
}

\author{
F. W. GEHRING, C. MACLACHLAN, G. J. MARTIN, AND A. W. REID
}

\begin{abstract}
We give an arithmetic criterion which is sufficient to imply the discreteness of various two-generator subgroups of PSL $(2, \mathbf{c})$. We then examine certain two-generator groups which arise as extremals in various geometric problems in the theory of Kleinian groups, in particular those encountered in efforts to determine the smallest co-volume, the Margulis constant and the minimal distance between elliptic axes. We establish the discreteness and arithmeticity of a number of these extremal groups, the associated minimal volume arithmetic group in the commensurability class and we study whether or not the axis of a generator is simple. We then list all "small" discrete groups generated by elliptics of order 2 and $n, n=3,4,5,6,7$.
\end{abstract}

\section{INTRODUCTION}

This paper is concerned with the geometry of Kleinian groups and hence the geometry and topology of hyperbolic 3-manifolds and orbifolds; see [4, 32, 33, 41] as basic references.

In $[18,21]$ we initiated a program to describe the analytic space of discrete two-generator subgroups of $\operatorname{PSL}(2, \mathbf{C})$ and developed a disk covering procedure to identify all two-generator Kleinian groups with elliptic generators. This method allowed us to identify many groups in [16] through [23] as extremals for various geometric problems or as finite index subgroups of extremal groups.

A principal obstruction encountered in this program, and hence to solving related geometric problems, was in establishing the discreteness of candidates identified by this procedure. In several cases we were able to accomplish this by means of an ad hoc method; see [18].

A main result of this paper gives a unified method, derived from algebraic number theory, which deals with many of these situations. As a consequence we find that many of the extremals are subgroups of arithmetic Kleinian or Fuchsian groups. These results are derived from a classical result in number theory whose application to proving groups discrete in $\operatorname{PSL}(2, \mathbf{C})$ seems to have been overlooked. When one considers how difficult it is in general to prove that a subgroup of $\operatorname{PSL}(2, \mathbf{C})$ is discrete, the criteria considered here seems surprisingly straightforward and elegant.

Received by the editors January 4, 1996.

1991 Mathematics Subject Classification. Primary 30F40, 20H10, 57N10.

Research supported in part by grants from the U. S. National Science Foundation, the N.Z. Foundation of Research, Science and Technology, the Australian Research Council, the U.K. Royal Society and the U.K. Scientific and Engineering Research Council. We wish also to thank the University of Texas at Austin and the University of Auckland for their hospitality during part of this work. We are grateful to D. J. Lewis who gave us a proof for Theorem 5.12 and to K. N. Jones who computed the co-volumes that appear in Table 11.

(c)XXXX American Mathematical Society 
The disk covering procedure mentioned above is based on complex iteration and a special semigroup of polynomials. It is ideally suited for the discreteness criteria which we shall establish here. Hence we first outline this procedure in $\S 2$ in order to motivate the criteria which we then give and discuss at the end of $\S 2 ; \S 3, \S 4$ and $\S 5$ contain background material on arithmetic groups and the proofs for the results given in $\S 2$

In $\S 6$ we apply these criteria to a family of two-generator groups $\langle f, g\rangle$ where $f$ and $g$ are elliptics of orders $n$ and $2, n=3,4,5,6,7$. These groups resulted from the disk covering procedure while studying the possible distances between non-intersecting elliptic axes in a discrete group .

The groups $\langle f, g\rangle$ in $\S 6$ are subgroups of arithmetic groups. In $\S 7$ and $\S 8$ we first compare the minimal co-volume of the arithmetic groups in which the groups $\langle f, g\rangle$ imbed with the distance between the axes of the generators $f$ and $g$. Using a computer program developed by $\mathrm{K}$. Jones and the last author it is possible to determine which of the groups $\langle f, g\rangle$ have finite co-volume and hence are themselves arithmetic.

Motivated by the small volume problem for orbifolds, we derive in $\S 9$ and then apply in $\S 10$ arithmetic and geometric criteria to determine which of the groups $\langle f, g\rangle$ of $\S 6$ have $f$ as a simple elliptic generator.

Finally we take a moment to indicate some of the more important applications of the results we obtain, in particular, of the existence and properties of the groups listed in Tables 1 through 12.

1. Minimal volume 3-orbifold. The minimal co-volume Kleinian group is not torsion free and does not contain any elliptic elements of order $n \geq 6$ $[23,24]$. Since the two-generator groups in Tables $6-10$ are listed in order of the distance between the axes of the generator $f$ and its nearest conjugate, these tables show that a low order torsion element either lies in an arithmetic subgroup or has a large precisely invariant collar. Table 12 can then be used to show that the minimal co-volume Kleinian group contains no simple 4 or 5 -torsion and at most one conjugacy class of simple 3-torsion [25].

2. Structure of the singular set. Tables 6-10 give sharp bounds for the distance between components of the singular set of a hyperbolic 3-orbifold and the distance between tetraheral, octahedral and icosahedral points in a Kleinian group; see also [12]. These results are used to show that the minimal volume 3 -fold has a very simple singular set or that the uniformizing Kleinian group is $G_{3,3}$ in Table $6[19,24,25]$.

3. Automorphism groups of 3-manifolds The above yield bounds for the order of the automorphism groups of many hyperbolic 3-manifold groups analogous to the $84(g-1)$ Theorem of Hurwitz. For example, if the automorphism group contains an element of order $n \geq 4$, then we can give sharp estimates on the order of the group.

4. Minimal volume 3-manifold. A recent computer search [15] motivated by the rigidity theorem in [14] has led people to ask if current estimates for the collar radius of a loxodromic with a short translation length can be significantly improved. Tables 1 and 2 contain examples which show that this is not possible without additional topological hypotheses such as in [11].

5. Margulis constant. The Margulis constant is achieved in a two- or threegenerator group. The groups given here realize this constant in the setting of 
two-generator groups with an elliptic generator as well as subgroups of those realizing the three-generator constant [20].

6. Geodesic length spectrum of 3-folds. Table 1 contains examples which show that current bounds on the length of intersecting closed geodesics, or nonsimple geodesics, are within a factor of 2 of being sharp. These, together with estimates on the Margulis constant, yield good bounds for the thick and thin decompositions.

\section{Disk COVERING METHOD AND DISCRETENESS}

Let $G=\langle f, g\rangle$ be a two-generator subgroup of $\operatorname{PSL}(2, \mathbf{C})$. We associate to each such group three complex numbers called the parameters of $G$,

$$
\operatorname{par}(G)=(\gamma(f, g), \beta(f), \beta(g)) \in \mathbf{C}^{3},
$$

where

$$
\beta(f)=\operatorname{tr}^{2}(f)-4, \beta(g)=\operatorname{tr}^{2}(g)-4, \gamma(f, g)=\operatorname{tr}([f, g])-2
$$

and $[f, g]=f g f^{-1} g^{-1}$ is the multiplicative commutator. These three complex numbers determine $G$ uniquely up to conjugacy whenever $\gamma(f, g) \neq 0$; recall $\gamma(f, g)=0$ implies the existence of a common fixed point. Conversely, every such triple determines a two-generator subgroup of $\operatorname{PSL}(2, \mathbf{C})$.

When $\gamma \neq 0, \beta$, there is a natural projection from $\mathbf{C}^{3}$ to $\mathbf{C}^{2}$, given by

$$
\left(\gamma, \beta, \beta^{\prime}\right) \rightarrow(\gamma, \beta,-4)
$$

which preserves discreteness; see [18]. That is, if $\left(\gamma, \beta, \beta^{\prime}\right)$ are the parameters for a discrete two-generator group, then so are $(\gamma, \beta,-4)$. Hence in these circumstances one can always replace one of the generators in a discrete two-generator group by an element of order two without altering the commutator or discreteness. No such result holds for groups with more than two-generators [7].

We shall study the pairs of parameters $(\gamma(f, g), \beta(f))$ in $\mathbf{C}^{2}$ which can correspond to discrete groups $\langle f, g\rangle$. For the purposes of this discussion we specialize to the case where $f$ is a primitive elliptic element of order $n \geq 3$. Then

$$
\beta=\beta(f)=-4 \sin ^{2}(\pi / n)
$$

and for each $n \geq 3$, we want to describe the set $E_{n}$ of values in the complex plane which may be assumed by the commutator parameter $\gamma(f, g)$ in the case when $\langle f, g\rangle$ is discrete. This set cannot have 0 as a limit point [18].

Next if

$$
h=g \circ f^{m_{1}} \circ g^{-1} \circ f^{m_{2}} \circ g \ldots f^{m_{n}} \circ g^{\epsilon}
$$

where $\epsilon=(-1)^{n}$, then it follows from [18] that

$$
\gamma(f, h)=p(\gamma(f, g), \beta(f))
$$

where $p$ is a polynomial in the two variables with integer coefficients. Equation (2.3) shows that for such words $h$, the value of $\gamma(f, h)$ depends only on $\gamma(f, g)$ and $\beta(f)$ and not on $\beta(g)$. In particular, the family of such polynomials $p$ is closed under composition in the first variable and so forms a polynomial semigroup $\mathbf{P}$. Thus we may study the iterates of a specific polynomial and, in this way, generate a sequence of commutator traces which cannot accumulate at 0 .

For example if $h=g f g^{-1} f g$ and $\gamma=\gamma(f, g)$, then

$$
p(\gamma, \beta)=\gamma(1+\beta-\gamma)^{2} .
$$


Hence if $f$ is elliptic of order 6 , then $\beta=-1$ and $p(\gamma, \beta)=\gamma^{3}$. In this case if we set $\gamma_{j}=\gamma\left(f, g_{j}\right)$ where

$$
g_{j+1}=g_{j} f g_{j}^{-1} f g_{j} \text { and } g_{1}=g
$$

then $\gamma_{j+1}=\gamma^{3^{j}} \rightarrow 0$ as $j \rightarrow \infty$ if $0<|\gamma|<1$, a contradiction. Thus $\gamma=0$ or $|\gamma| \geq 1$ and we obtain an analogue for elliptics of order 6 of the classical inequality of Shimizu-Leutbecher which yields the same conclusion whenever $f$ is parabolic [33]. In particular,

where $D_{6}=\{z: 0<|z|<\mid\}$.

$$
E_{6} \subset \mathbf{C} \backslash D_{6}
$$

For each value of $n \geq 3$ we can argue as above using iteration with an appropriate polynomial in $\mathbf{P}$ to obtain an open punctured disk $D_{n}$ about 0 which does not contain the parameter $\gamma(f, g)$. When $n \geq 7$, the existence of such a neighborhood follows from Jørgensen's inequality. Next if $p(\gamma)=p(\gamma, \beta)$ is the polynomial in $\mathbf{P}$ which corresponds to the element $h$ in $(2.2)$, then the subgroup $\langle f, h\rangle$ cannot be discrete if

$$
p(\gamma(f, g))=p(\gamma(f, g), \beta(f))=\gamma(f, h) \in D_{n} .
$$

Hence $p^{-1}\left(D_{n}\right)$ is an excluded region for $\gamma(f, g)$ and we conclude that

$$
E_{n} \subset \mathbf{C} \backslash\left\{\bigcup p^{-1}\left(D_{n}\right): p \in \mathbf{P}\right\} .
$$

In this way we can cover regions of the complement of the set $E_{n}$ of admissible values for the commutator parameter of groups with a generator of order $n$.

The program for describing these regions was begun in [18] and was significantly extended in [21]. Obtaining various geometric constraints for such groups amounts to showing that a certain region $\Omega$ lies in the complement of $E_{n}$ except possibly for some exceptional values.

The result of this disk covering argument is illustrated below for the case where $n=3$ (see Figure 1). All exceptional values within the region covered by the disks are illustrated; they are the only possible values for the commutator parameter of a discrete group which lies in the union of these disks. In this case it turns out that every value corresponds to a subgroup of an arithmetic group. Many are in fact two-generator arithmetic groups themselves. That is, they are additionally of finite co-volume.

The closure of the hyperbolic line joining the two fixed points of a non-parabolic element $f$ is called the axis of $f$, denoted by axis $(f)$. The following formula yields collaring theorems from which volume estimates may be obtained; see [19] and [23].

Lemma 2.4. If $f$ and $g$ are non-parabolic, then the hyperbolic distance $\delta(f, g)$ between the axes of $f$ and $g$ is given by

$$
\cosh (2 \delta(f, g))=\left|\frac{4 \gamma(f, g)}{\beta(f) \beta(g)}+1\right|+\left|\frac{4 \gamma(f, g)}{\beta(f) \beta(g)}\right| .
$$

The part of $E_{n}$ in $\Omega$ is contained in the union $X_{n}$ of the preimages of the punctures of $D_{n}$ taken over all the polynomials $p$ in $\mathbf{P}$. Moreover $E_{n}$ is a proper subset of $X_{n}$ since the preimage of a puncture under one polynomial $p$ in $\mathbf{P}$ can often be mapped into $D_{n}$ by some other polynomial $q$ in $\mathbf{P}$, and hence will not be a point in $E_{n}$. At the end of such a covering argument one is left with a few points of $X_{n}$ inside the given region $\Omega$ which may correspond to discrete groups. One must then analyze these exceptions and prove that either they are not discrete or, if they 


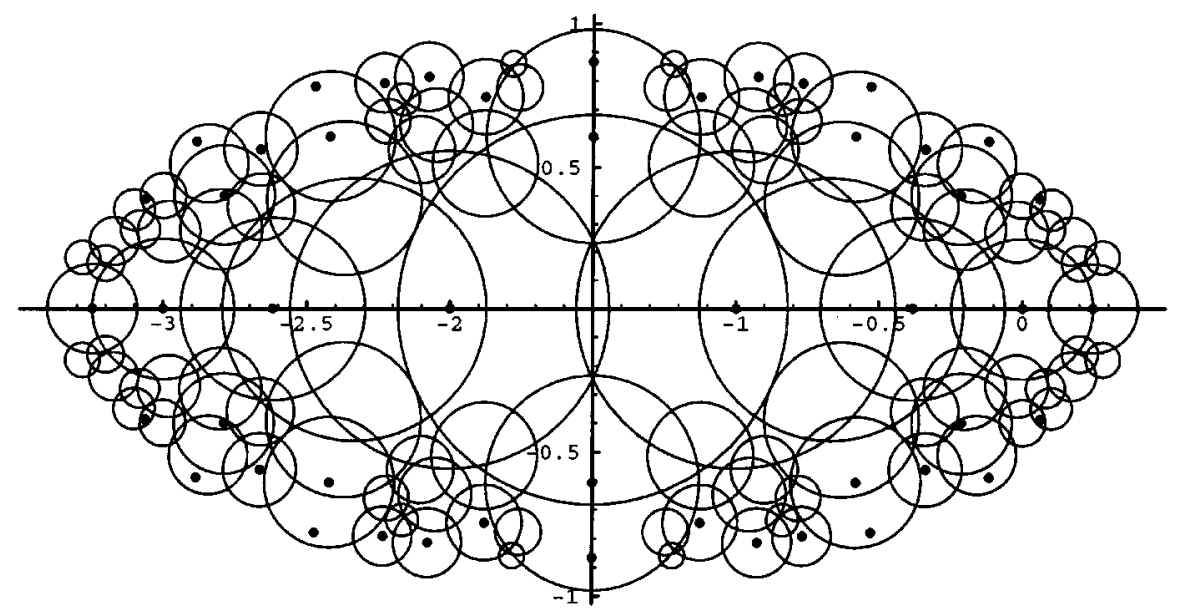

Figure 1. Possible values for commutator parameter when $n=3$

are discrete, use knowledge of these exceptional groups to compute the invariants being studied.

It is often not easy to decide whether a given point $\gamma$ in $X_{n}$ belongs to $E_{n}$, i.e. whether $\gamma$ corresponds to discrete group with a generator of order $n$. However in our situation there exists a certain polynomial with coefficients in $\mathbf{Z}[\beta]$ for which $\gamma$ is a root. Thus it is natural to look for arithmetic conditions which guarantee discreteness. The fortunate fact is that this works well for the small extremals, the general philosophy being that "small" implies "highly symmetric" which in turn implies "arithmetic".

Under the assumptions of discreteness and finite co-volume on subgroups of $\operatorname{PSL}(2, \mathbf{R})$ and $\operatorname{PSL}(2, \mathbf{C})$, the results of $[40]$ and [30] give conditions on the traces of elements in the subgroup which characterize, among Fuchsian and Kleinian groups, those which are arithmetic; see $\S 4$. Dropping the assumption of finite co-volume characterizes those which are subgroups of arithmetic Fuchsian or Kleinian groups. More importantly, the proofs in [40] and [30] make no use of the discreteness assumption; cf. [3]. Thus the arithmetic conditions on the traces can be used to deduce discreteness.

In this paper refinements of these arithmetic conditions which will guarantee discreteness are obtained. These are particularly applicable to two-generator groups such as those corresponding to the points $\gamma$ in $X_{n}$ described above.

For example, suppose that $G=\langle f, g\rangle$ where $f$ is a primitive elliptic element of order $n \geq 3$ and $g$ is elliptic of order 2 . Then

$$
\beta=\beta(f)=-4 \sin ^{2}(\pi / n)
$$

is an algebraic integer in the field $\mathbf{Q}(\cos (2 \pi / n))$, which is totally real with $\phi(n) / 2$ places. Let $R_{n}$ denote the ring of integers in $\mathbf{Q}(\cos (2 \pi / n))$ and note that $R_{n}=\mathbf{Z}[\beta]$. The Galois conjugates of $\beta, \sigma(\beta)=-4 \sin ^{2}(m \pi / n)$ where $(m, n)=1$, lie in the interval $(-4,0)$ with $\sigma(\beta) \leq \beta$. Recall that $G$ is determined up to conjugacy by $\beta$ and $\gamma(f, g)$. With this we prove the following criteria for discreteness. Recall that, for a number field $K$ of degree $n$ over $\mathbf{Q}$, the $n$ Galois embeddings $\sigma: K \rightarrow \mathbf{C}$ give 
rise to valuations on $K$ which fall into equivalence classes - the places-modulo the action of complex conjugation.

Theorem 2.6. Let $G=\langle f, g\rangle$ be a subgroup of $\operatorname{PSL}(2, \mathbf{C})$ with $f$ a primitive elliptic element of order $n \geq 3, g$ an elliptic of order 2 and $\gamma(f, g) \neq 0, \beta(f)$. Then $G$ is a subgroup of a discrete arithmetic group if

1. $\mathbf{Q}(\gamma(f, g), \beta(f))$ has at most one complex place;

2. $\gamma(f, g)$ is a root of a monic polynomial $p(z) \in \mathbf{Z}[\beta][z]$;

3. if $\gamma(f, g)$ and $\bar{\gamma}(f, g)$ are not real, then all other roots of $p(z)$ are real and lie in the interval $(\beta, 0)$;

4. if $\gamma(f, g)$ is real, then all other roots of $p(z)$ are real and lie in the interval $(\beta, 0)$.

The requirement that the field $\mathbf{Q}(\gamma(f, g), \beta(f))$ has at most one complex place can, for $\beta(f)$ as in Theorem 2.6, also be described in terms of the polynomial $p(z)$. If in addition $n=3,4$ or 6 , then $\beta \in \mathbf{Z}$ and the criteria admit a greatly simplified description. See Theorem 5.14 below.

\section{A CRITERION FOR DISCRETENESS}

In this section we prove a result that guarantees discreteness under certain conditions on the invariant trace field. Our result is related to a classical result in number theory and does not seem to be well known in the area of Kleinian groups. Indeed it is a generalization of the prototype result that yields discreteness for arithmetic subgroups of $\operatorname{PSL}(2, \mathbf{C})$. As we point out in [29] this theorem has a proof in the language of arithmetic groups which makes application more routine for our purposes; see also the discussion in $\S 4$. However we give here an elementary proof which illuminates the connection with the following well known facts from number theory.

Recall that if $p$ is a monic irreducible polynomial over $\mathbf{Z}$ of degree $n$ with roots $\alpha_{1}, \ldots, \alpha_{n}$, then

$$
p(z)=\prod_{i=1}^{n}\left(z-\alpha_{i}\right)=z^{n}-s_{1} z^{n-1}+\ldots+(-1)^{k} s_{k} z^{n-k}+\cdots+(-1)^{n} s_{n}
$$

where $s_{i}$ is the $i^{\text {th }}$ symmetric polynomial in $\alpha_{1}, \ldots, \alpha_{n}$. As a consequence we deduce the following easy lemma.

Lemma 3.2. There are only finitely many algebraic integers $z$ of bounded degree such that $z$ and all Galois conjugates of $z$ are bounded.

Proof. Let $z$ have degree $n$ over $\mathbf{Q}$. If $z$ and its Galois conjugates are bounded, then the coefficients of the irreducible polynomial $p$ of $z$ are symmetric polynomials in the roots of $p$ and hence are bounded. Therefore only finitely many integers can arise as coefficients of such a polynomial.

Next we recall some notation from [35]. Let $G$ be a finitely generated subgroup of $\operatorname{PSL}(2, \mathbf{C})$. The trace field of $G$ is the field generated over $\mathbf{Q}$ by the set

$$
\operatorname{tr}(G)=\{ \pm \operatorname{tr}(g): g \in G\} .
$$


Since $G$ is finitely generated, the subgroup $G^{(2)}=\left\langle g^{2}: g \in G\right\rangle$ is a normal subgroup of finite index with quotient group a finite abelian 2-group. Following [35] we call

$$
k G=\mathbf{Q}\left(\operatorname{tr}\left(G^{(2)}\right)\right)
$$

the invariant trace-field of $G$. For any finite index subgroup $G_{1}$ of a non-elementary group $G$ one can show that $\mathbf{Q}\left(\operatorname{tr}\left(G^{(2)}\right)\right) \subset \mathbf{Q}\left(\operatorname{tr}\left(G_{1}\right)\right)$; in [38] it is shown that $k G$ is an invariant of the commensurability class.

Throughout the paper, we will use

$$
\mathbf{c}: \mathbf{C} \rightarrow \mathbf{C}
$$

to denote the complex conjugation map. With this notation we can establish the following criterion for discreteness.

Theorem 3.4. Let $G$ be a finitely generated subgroup of $\operatorname{PSL}(2, \mathbf{C})$ such that

1. $G^{(2)}$ contains elements $g_{1}$ and $g_{2}$ which have no common fixed point;

2. $\operatorname{tr}(G)$ consists of algebraic integers;

3. for each embedding $\sigma: k G \rightarrow \mathbf{C}$ such that $\sigma \neq i d$ or $\mathbf{c}$, the set $\{\sigma(\operatorname{tr}(f)): f \in$ $\left.G^{(2)}\right\}$ is bounded.

Then $G$ is discrete.

Proof. Note that since $G$ is finitely generated, so is $G^{(2)}$ and so all traces in $G^{(2)}$ are obtained from integral polynomials in a finite number of traces. Thus $k G$ is a finite extension of $\mathbf{Q}$.

It suffices to prove that the finite index subgroup $G^{(2)}$ is discrete. Suppose that this is not the case and let $f_{n}$ be a sequence of distinct elements converging to the identity in $G^{(2)}$. If $z_{n}=\operatorname{tr}\left(f_{n}\right)$ and $z_{n, i}=\operatorname{tr}\left(\left[f_{n}, g_{i}\right]\right)$, then

$$
\beta\left(f_{n}\right)=z_{n}^{2}-4 \rightarrow 0 \text { and } \gamma\left(f_{n}, g_{i}\right)=z_{n, i}-2 \rightarrow 0
$$

for $i=1,2$ as $n \rightarrow \infty$. Hence we may assume that $\left|z_{n}\right|<K$ for some fixed constant $K$. Next by condition $3,\left|\sigma\left(z_{n}\right)\right|<K_{\sigma}$ for each embedding $\sigma \neq i d$ or $\mathbf{c}$ of $k G$, where $K_{\sigma}$ is a constant which depends only on $\sigma$.

Let $R=\max \left\{K, K_{\sigma}\right\}$ where $\sigma$ ranges over all embeddings $\sigma \neq i d$ or $\mathbf{c}$ of $k G$. Then the algebraic integers $z_{n}$ are of bounded degree and they and all their Galois conjugates are bounded in absolute value by $R$. By Lemma 3.2, the $z_{n}$ assume only finitely many values. Thus for large $n, \beta\left(f_{n}\right)=0$ and $f_{n}$ is parabolic with a single fixed point $w_{n}$.

Next we can apply the above argument to the algebraic integers $z_{n, i}$ to conclude that $\gamma\left(f_{n}, g_{i}\right)=0$ for $i=1,2$ and large $n$. This then implies that $g_{1}$ and $g_{2}$ each have $w_{n}$ as a common fixed point for large $n$ contradicting condition 1 .

\section{Arithmetic Kleinian groups}

In this section we first recall some terminology about quaternion algebras and then discuss Theorem 3.4 in the language of these algebras and arithmetic groups. In particular we give a version of Theorem 3.4 which is readily applicable, especially in the context of two-generator groups; see $\S 5$.

We begin with some facts about quaternion algebras; see [42] for details. Let $k$ be a number field, let $\nu$ be a place of $k$, i.e. an equivalence class of valuations on $k$ and denote by $k_{\nu}$ the completion of $k$ at $\nu$. If $B$ is a quaternion algebra over $k$, we say that $B$ is ramified at $\nu$ if $B \otimes_{k} k_{\nu}$ is a division algebra of quaternions. Otherwise $\nu$ is unramified. 
In case $\nu$ is a place associated to a real embedding of $k, B$ is ramified if and only if $B \otimes_{k} k_{\nu} \cong \mathcal{H}$, where $\mathcal{H}$ is the Hamiltonian division algebra of quaternions.

It is straightforward to check whether a quaternion algebra is ramified at a real place. Recall, following [42], the Hilbert symbol $\left(\frac{a, b}{k}\right)$ corresponding to the quaternion algebra $\{1, i, j, i j\}$ defined over the field $k$ with $i^{2}=a, j^{2}=b$ and $i j=-j i$.

Lemma 4.1. Let $B=\left(\frac{a, b}{k}\right)$. Then $B$ is ramified at a real place $\nu$ corresponding to the real embedding $\sigma$ if and only if $\sigma(a)$ and $\sigma(b)$ are negative.

Proof. By definition, $B$ is ramified at $\nu$ whenever $B \otimes_{k} k_{\nu} \cong \mathcal{H}$. This tensor product is isomorphic to

$$
\left(\frac{\sigma(a), \sigma(b)}{\sigma(k)}\right) \otimes_{\sigma(k)} \mathbf{R}
$$

or simply $\left(\frac{\sigma(a), \sigma(b)}{\mathbf{R}}\right)$. This, in turn, is isomorphic to $\mathcal{H}$ exactly when $\sigma(a)$ and $\sigma(b)$ are negative since one can remove squares without affecting the isomorphism class of the quaternion algebra; cf. [42].

We now give the definition of an arithmetic Kleinian group. Let $k$ be a number field with one complex place and $A$ a quaternion algebra over $k$ ramified at all real places. Next let $\rho$ be an embedding of $A$ into $M(2, \mathbf{C}), \mathcal{O}$ an order of $A$ and $\mathcal{O}^{1}$ the elements of norm 1 in $\mathcal{O}$. Then $\rho\left(\mathcal{O}^{1}\right)$ is a discrete subgroup of $\operatorname{SL}(2, \mathbf{C})$ and its projection to $\operatorname{PSL}(2, \mathbf{C}), P \rho\left(\mathcal{O}^{1}\right)$, is an arithmetic Kleinian group. The commensurability classes of arithmetic Kleinian groups are obtained by considering all such $P \rho\left(\mathcal{O}^{1}\right)$.

Arithmetic Fuchsian groups arise in a similar manner. In this case the number field is totally real and the algebra ramified at all real places except the identity.

In [30] (resp. [40]), it is shown that two arithmetic Kleinian groups (resp. arithmetic Fuchsian groups) are commensurable up to conjugacy if and only if their invariant quaternion algebras are isomorphic; see also [5].

To state the characterization theorems in [30] and [40] referred to earlier, we first define, following Bass [3],

$$
A G=\left\{\sum a_{i} g_{i}: a_{i} \in \mathbf{Q}(\operatorname{tr}(G)), \quad g_{i} \in G\right\}
$$

for any finitely generated non-elementary subgroup of $\operatorname{SL}(2, \mathbf{C})$. Then $A G$ is a quaternion algebra over $\mathbf{Q}(\operatorname{tr}(G))$. Recall that $k G=\mathbf{Q}\left(\operatorname{tr}\left(G^{(2)}\right)\right)$ and $A G^{(2)}$ is then the invariant quaternion algebra since, for a finitely generated non-elementary subgroup $G$ of $\operatorname{PSL}(2, \mathbf{C})$, the pair $\left(A G^{(2)}, k G\right)$ is an invariant of the commensurability class of $G$. See [35] and also [43] where such things are implicitly discussed. Additionally, if $\operatorname{tr}(G)$ consists of algebraic integers, then as in [3]

$$
\mathcal{O} G=\left\{\sum a_{i} g_{i} \mid a_{i} \in R_{\mathbf{Q}(\operatorname{tr}(G))}, \quad g_{i} \in G\right\}
$$

is an order in $A G$, where $R_{\mathbf{Q}(\operatorname{tr}(G))}$ denotes the ring of integers in $\mathbf{Q}(\operatorname{tr}(G))$.

Theorem 4.2. Let $G$ be a Kleinian (resp. Fuchsian) group of finite co-volume. Then $G$ is arithmetic if and only if the following conditions are satisfied:

1. $k G$ is an algebraic number field;

2. $\operatorname{tr}(G)$ consists of algebraic integers;

3. for every Q-isomorphism $\sigma: k G \rightarrow \mathbf{C}$ such that $\sigma \neq i d, \mathbf{c}, \sigma\left(\operatorname{tr}\left(G^{(2)}\right)\right)$ is bounded in $\mathbf{C}$. 
Sketch of Proof. It is a simple matter to show that arithmetic Kleinian and Fuchsian groups have these properties. Note that if $\gamma \in \mathcal{H}^{1} \cong S^{3}$, then $\operatorname{tr}(\gamma) \in[-2,2]$.

Since $G$ has finite co-volume, it is finitely generated and non-elementary. Only these two properties are used in the proof, except at the final stage. Condition 1 actually follows from 2 and the fact that $G$ is finitely generated. We thus obtain a quaternion algebra $A G^{(2)}$ over $k G$ with $A G^{(2)} \subset M(2, \mathbf{C})$. Furthermore, each embedding $\sigma: k G \rightarrow \mathbf{C}$ extends to an embedding of $A G^{(2)}$ in $M(2, \mathbf{C})$.

If $\gamma \in \mathrm{SL}(2, \mathbf{C})$ has eigenvalues $u, u^{-1}$ whose absolute values are not one, then it follows that $\left\{\operatorname{tr}\left(\gamma^{m}\right): m \in \mathbf{Z}\right\}$ is unbounded since

$$
\left|\operatorname{tr}\left(\gamma^{m}\right)\right| \geq\left.|| u\right|^{m}-|u|^{-m} \mid .
$$

Thus in the case at hand condition 3 implies that $k G$ has exactly one complex place if $k G$ is not real and is totally real if $k G$ is real. Also with $\gamma$ as above, $\operatorname{tr}(\gamma) \in[-2,2]$ and condition 3 also implies that $A G^{(2)}$ is ramified at all real places of $k G \neq i d$. Finally condition 2 shows that $\mathcal{O} G^{(2)}$ is an order of $A G^{(2)}$ and, of course, $G^{(2)} \subset\left(\mathcal{O} G^{(2)}\right)^{1}$, which is a discrete arithmetic Kleinian or Fuchsian group. Since $G$ has finite co-volume, it will be commensurable with $\left(\mathcal{O} G^{(2)}\right)^{1}$ and so be arithmetic.

In practice, direct application of Theorem 4.2 (or Theorem 3.4) is hard, the problem being to establish the boundedness of the traces at real embeddings. However, as a corollary of the proof of Theorem 4.2, we obtain the following more useful method for proving groups discrete.

Theorem 4.3. Let $G$ be a finitely generated non-elementary subgroup of $\operatorname{PSL}(2, \mathbf{C})$ such that

1. $k G$ has exactly one complex place or is totally real;

2. $\operatorname{tr}(G)$ consists of algebraic integers;

3. $A G^{(2)}$ is ramified at all places of $k G, \neq\{i d, \mathbf{c}\}$.

Then $G$ is a subgroup of an arithmetic Kleinian or Fuchsian group.

If $g_{1}, g_{2} \in G^{(2)}$ have no common fixed point and $g_{1}$ is not parabolic, the proof of Theorem 4.2 shows that $A G^{(2)}$ is spanned by $1, g_{1}, g_{2}, g_{1} g_{2}$ over $k G$; see [27], [30] and [40]. A basis in standard form can then be obtained yielding the Hilbert symbol

$$
\left(\frac{\beta\left(g_{1}\right), \gamma\left(g_{1}, g_{2}\right)}{k G}\right)
$$

Now if $f, g \in G$ are a pair of non-commuting elements with $f$ not parabolic and $f, g$ not of order 2 , then

$$
\beta\left(f^{2}\right)=(\beta(f)+4) \beta(f), \quad \gamma\left(f^{2}, g^{2}\right)=(\beta(f)+4)(\beta(g)+4) \gamma(f, g)
$$

so that

$$
A G^{(2)} \cong\left(\frac{(\beta(f)+4) \beta(f),(\beta(f)+4)(\beta(g)+4) \gamma(f, g)}{k G}\right) .
$$

See [27]. If we choose, as we may do, $f$ and $g$ to have equal traces, then by removing squares in the Hilbert symbol we obtain

$$
A G^{(2)} \cong\left(\frac{(\beta(f)+4) \beta(f), \gamma(f, g)}{k G}\right) .
$$


Finally note that if $k G=\mathbf{Q}(\operatorname{tr}(G))$ we can simplify still further to

$$
A G^{(2)} \cong\left(\frac{\beta(f), \gamma(f, g)}{k G}\right) \text {. }
$$

With these representations of $A G^{(2)}$, condition 3 of Theorem 4.3 can be readily checked using Lemma 4.1. Thus in summary we have the following result.

Theorem 4.7. Let $G$ be a non-elementary finitely generated subgroup of $\operatorname{PSL}(2, \mathbf{C})$ with a pair of non-commuting elements $f$ and $g$, where $f$ is not parabolic and $f$ and $g$ are both not of order 2. Then $G$ is discrete if

1. $\mathbf{Q}(\operatorname{tr}(G))$ is a finite extension of $\mathbf{Q}$;

2. $\operatorname{tr}(G)$ consists of algebraic integers;

3. $k G$ has one complex place or is totally real;

4. with $A G^{(2)} \cong\left(\frac{a, b}{k G}\right)$ described at (4.4), (4.5), (4.6), then $\sigma(a)$ and $\sigma(b)$ are negative for each embedding $\sigma$ of $k G \neq i d$, c.

\section{TWO-GENERATOR GROUPS}

Here we specialize the discreteness theorems in the previous sections to the case where $G$ is a two-generator group and, in particular, where both generators are elliptic. In these cases, both the invariant field and the invariant quaternion algebra will be readily described in terms of the parameters of the group.

As stated above, $k G=\mathbf{Q}\left(\operatorname{tr}\left(G^{(2)}\right)\right)$ is an invariant of the commensurability class. In fact, it is shown in [38] that the field $k G$ coincides with the field

$$
\mathbf{Q}\left(\left\{\operatorname{tr}^{2}(g): g \in G\right\}\right)=\mathbf{Q}(\{\beta(g): g \in G\}) .
$$

Actually, it is not difficult to see that if $G$ is generated by elements $g_{1}, g_{2}, \ldots, g_{n}$ with $\beta\left(g_{i}\right) \neq-4$ for $i=1,2, \ldots, n$, then

$$
k G=\mathbf{Q}\left(\operatorname{tr}\left(G^{s q}\right)\right)
$$

where $G^{s q}=\left\langle g_{1}^{2}, g_{2}^{2}, \ldots, g_{n}^{2}\right\rangle$; see [27]. For two-generator groups this has the following consequence.

Lemma 5.2. Let $G=\langle f, g\rangle$ be a subgroup of $\mathrm{SL}(2, \mathbf{C})$ with $\beta(f) \neq-4$ and $\beta(g) \neq$ -4 . Then

$$
k G=\mathbf{Q}\left(\beta(f), \beta(g), \beta\left(f g^{-1}\right)-\gamma(f, g)\right) .
$$

Proof. The trace of any element in a two-generator group $\langle\phi, \psi\rangle$ group is given by a polynomial with integer coefficients in $\operatorname{tr}(\phi), \operatorname{tr}(\psi), \operatorname{tr}\left(\phi \psi^{-1}\right)$. See, for example [28]. Thus $\mathbf{Q}\left(\operatorname{tr}\left(G^{s q}\right)\right)$ is $\mathbf{Q}\left(\operatorname{tr}\left(f^{2}\right), \operatorname{tr}\left(g^{2}\right), \operatorname{tr}\left(f^{2} g^{-2}\right)\right)$. Then since

$$
\operatorname{tr}\left(f^{2}\right)=\beta(f)+2, \quad \operatorname{tr}\left(g^{2}\right)=\beta(g)+2,
$$

the desired conclusion follows from the identity

$$
\operatorname{tr}\left(f^{2} g^{-2}\right)=\beta\left(f g^{-1}\right)+2-\gamma(f, g) .
$$

The following corollary will be of use to us.

Corollary 5.4. Let $G=\langle f, g\rangle$ be a group with $\beta(f) \neq-4$ and $\gamma(f, g) \neq 0, \beta(f)$. If $G_{1}=\left\langle f, g f g^{-1}\right\rangle$, then

$$
k G_{1}=\mathbf{Q}(\beta(f), \gamma(f, g)) .
$$


Proof. Applying Lemma 5.2 in the case where the generators are $f$ and $g f g^{-1}$ and $f$ as above, we see that

$$
k G_{1}=\mathbf{Q}\left(\beta(f), \beta\left(f g f^{-1} g^{-1}\right)-\gamma\left(f, g f g^{-1}\right)\right) .
$$

An easy calculation shows

$$
\beta\left(f g f^{-1} g^{-1}\right)-\gamma\left(f, g f g^{-1}\right)=\gamma(f, g)(\beta(f)+4) .
$$

Next the hypotheses on $\beta(f)$ and $\gamma(f, g)$ imply that $f$ and $g f g^{-1}$ do not commute. Thus $k G_{1}=\mathbf{Q}(\gamma(f, g), \beta(f))$.

Corollary 5.7. Let $G=\langle f, g\rangle$ be a group with $\beta(f) \neq-4, \beta(g)=-4$ and $\gamma(f, g) \neq 0, \beta(f)$. Then

$$
k G=\mathbf{Q}(\gamma(f, g), \beta(f)) .
$$

Proof. Since $g$ has order two, $G_{1}=\left\langle f, g f g^{-1}\right\rangle$ has index two in $\langle f, g\rangle$. Thus $k G_{1}=k G$ being an invariant of the commensurability class and the result follows from Corollary 5.4.

The hypotheses of Corollary 5.7 imply that the groups $G=\langle f, g\rangle$ and $G_{1}=$ $\left\langle f, g f g^{-1}\right\rangle$ are simultaneously discrete or nondiscrete. For two-generator groups $G$ we have also identified $k G$ in terms of the parameters of $G$. We have therefore proved the following result.

Corollary 5.8. Let $G=\langle f, g\rangle$ be a group with $\operatorname{par}(G)=(\gamma, \beta,-4)$ where $\beta \neq-4,0$ and $\gamma \neq 0, \beta$. Then $k G=\mathbf{Q}(\gamma, \beta)$ and

$$
A G^{(2)}=\left(\frac{\beta(\beta+4), \gamma(\gamma-\beta)}{\mathbf{Q}(\gamma, \beta)}\right) \text {. }
$$

Proof. Again $G_{1}=\left\langle f, g f g^{-1}\right\rangle$ is a subgroup of index two in $G$ with

$$
\operatorname{par}\left(G_{1}\right)=(\gamma(\gamma-\beta), \beta, \beta) .
$$

Our hypotheses imply this group is non-elementary and $k G_{1}=k G=\mathbf{Q}(\gamma, \beta)$. The Hilbert symbol follows from (4.5).

From Theorem 4.7, we can now deduce as a corollary the following sufficient condition for a two-generator group to be a subgroup of an arithmetic Kleinian group, and hence discrete.

Theorem 5.10. Let $G=\langle f, g\rangle$ be a subgroup of $\operatorname{PSL}(2, \mathbf{C})$ with $\operatorname{par}(G)=(\gamma, \beta,-4)$ where $\beta \neq 0$ and $\gamma \neq 0, \beta$. Then $G$ is a discrete group if

1. $\gamma$ and $\beta$ are algebraic integers;

2. the field $k G=\mathbf{Q}(\gamma, \beta)$ has exactly one complex place or is totally real;

3. if $k G$ has a complex place, then $-4<\sigma(\beta)<0$ and $\sigma(\gamma(\gamma-\beta))<0$ for all real embeddings $\sigma$;

4. if $k G$ is totally real, then $-4<\sigma(\beta)<0$ and $\sigma(\gamma(\gamma-\beta))<0$ for all real embeddings $\sigma \neq i d$.

Indeed $G$ is a subgroup of an arithmetic Fuchsian or Kleinian group with invariant trace field $\mathbf{Q}(\gamma, \beta)$ where the Hilbert symbol of the invariant quaternion algebra is

$$
\left(\frac{(\beta+4) \beta, \gamma(\gamma-\beta)}{\mathbf{Q}(\gamma, \beta)}\right)
$$


Proof. The traces of $f, f g$ satisfy monic quadratic polynomials whose coefficients lie in $\mathbf{Z}[\gamma, \beta]$. Since the traces of elements in $G$ are polynomials with integer coefficients in $\operatorname{tr}(f), \operatorname{tr}(g), \operatorname{tr}(f g)$, the first hypothesis implies that all traces of elements of $G$ are algebraic integers. Therefore the elements of $G^{(2)}$ also have algebraic integer traces. By Corollary 5.8, the Hilbert symbol for $A G^{(2)}$ is as claimed, and so by the last pair of assumptions we see that $A G^{(2)}$ is ramified at all real places, except possibly in the case when $k G$ is totally real.

By our previous discussion it follows that $\mathcal{O} G^{(2)}$ is an order of $A G^{(2)}$, and therefore $\mathcal{O} G^{(2)^{1}}$ yields an arithmetic Kleinian or Fuchsian group via the construction discussed previously. Thus $G^{(2)}$ is discrete and hence so is $G$.

In the proof we constructed the arithmetic group $\mathcal{O} G^{(2)^{1}}$ which contains $G^{(2)}$. To get an arithmetic group containing $G$ consider the group

$$
\operatorname{Norm}\left(\mathcal{O} G^{(2)}\right)=\left\{x \in A G^{(2)}: x \mathcal{O} G^{(2)} x^{-1}=\mathcal{O} G^{(2)}\right\} .
$$

The image of this group is an arithmetic subgroup of $\operatorname{PSL}(2, \mathbf{C})$ (see [5] for example). As $G^{(2)}$ is normal in $G$, it follows that $G$ is a subgroup of an arithmetic Kleinian group.

In the cases where $f$ is primitive elliptic of order $n$ and $g$ is elliptic of order 2 , then $\beta=-4 \sin ^{2} \pi / n$ is a totally real algebraic integer so $\gamma$ must satisfy a monic polynomial in $\mathbf{Z}[\beta][z]$ to be an algebraic integer. For all embeddings $\sigma$ of $\mathbf{Q}(\gamma, \beta)$, $-4<\sigma(\beta) \leq \beta<0$ and so Theorem 2.6 is an immediate corollary of Theorem 5.10.

We now refine these results to obtain more easily applicable criteria for discreteness. We first recall some basic results concerning field extensions.

Suppose that $\mathbf{K}$ is a finite extension of $\mathbf{Q}$, that $\gamma$ is algebraic over $\mathbf{K}$ and that $p(z)$ is the minimum polynomial of $\gamma$ over $\mathbf{K}$ with $\operatorname{deg}(p)=m>1$. Let $\sigma: \mathbf{K} \rightarrow \mathbf{C}$ be an embedding. Then there are exactly $m$ embeddings $\tau: \mathbf{K}(\gamma) \rightarrow \mathbf{C}$ such that $\tau \mid \mathbf{K}=\sigma$ and these are uniquely determined by $\tau(\gamma)=\gamma^{\prime}$ where $\gamma^{\prime}$ runs through the roots of $\sigma(p(z))$. See for example [13].

Lemma 5.11. If $\mathbf{K}(\gamma)$ has exactly one complex place, then $\mathbf{K}$ must be totally real, i.e. every embedding of $\mathbf{K}$ into $\mathbf{C}$ must be real.

Proof. If $\sigma: \mathbf{K} \rightarrow \mathbf{C}$ is a complex embedding, so is $\bar{\sigma}$ and $\bar{\sigma} \neq \sigma$. Then by the above remarks, there exist $2 m$ embeddings of $\mathbf{K}$ which are complex.

Theorem 5.12. Let $\gamma$ and $\beta$ be algebraic integers such that $\gamma \notin \mathbf{Q}(\beta) \subset \mathbf{Q}(\gamma, \beta)$ and let $p(z, \beta)$ be the minimum polynomial of $\gamma$ over $\mathbf{Q}(\beta)$. Then $\mathbf{Q}(\gamma, \beta)$ has one complex place if and only if

1. $\beta$ is totally real, i.e. all of the Galois conjugates of $\beta, \beta=\beta_{1}, \beta_{2}, \ldots, \beta_{n}$ are real;

2. the polynomial $q(z)=p\left(z, \beta_{1}\right) p\left(z, \beta_{2}\right) \cdots p\left(z, \beta_{n}\right)$ has exactly one pair of complex conjugate roots and all other roots real.

Proof. For $j=1,2, \ldots, n$ let $\sigma_{j}$ denote the embedding of $\mathbf{Q}(\beta)$ into $\mathbf{C}$ defined by $\sigma_{j}(\beta)=\beta_{j}$ and suppose that $\tau$ is an embedding of $\mathbf{Q}(\gamma, \beta)$ into $\mathbf{C}$. Then $\tau$ is determined by the images $\tau(\gamma), \tau(\beta)$. Now $\tau(\beta)=\sigma_{j}(\beta)$ for some $j$ in which case $\tau(\gamma)$ is a root of $\sigma_{j}(p(z, \beta))=p\left(z, \beta_{j}\right)$. Thus conditions 1 and 2 imply that there are precisely two complex embeddings $\mathbf{Q}(\gamma, \beta)$ into $\mathbf{C}$.

Conversely if $\mathbf{Q}(\gamma, \beta)$ has exactly one pair of complex embeddings into $\mathbf{C}$, then by Lemma 5.11 each embedding of $\mathbf{Q}(\beta)$ into $\mathbf{C}$ must be real and so an embedding 
$\tau$ of $\mathbf{Q}(\gamma, \beta)$ into $\mathbf{C}$ will be complex precisely when a root of $\sigma_{j}(p(z, \beta))=p\left(z, \beta_{j}\right)$ is complex.

Note that with $\gamma, \beta$ as in Theorem 5.12, $\mathbf{Q}(\gamma)$ must have at least one complex place and so by Lemma 5.11, $\mathbf{Q}(\gamma)=\mathbf{Q}(\gamma, \beta)$.

Now specialize again to the cases where $G=\langle f, g\rangle$ with $f$ a primitive elliptic element of order $n \geq 3, g$ elliptic of order 2 and $\gamma \neq 0, \beta$. Thus $\mathbf{Q}(\beta)$ is totally real and the Galois conjugates $\beta_{k}=-4 \sin ^{2}(k \pi / n)$ where $(k, n)=1$ and $1 \leq k \leq n / 2$ so that $\beta_{k}=\beta\left(f^{k}\right)$. Note that $-4<\beta_{k}<\beta<0$ for $k \neq 1$. Thus from Theorems 5.10 and 5.12, we obtain the following result.

Theorem 5.13. Let $G=\langle f, g\rangle$ be a subgroup of $\operatorname{PSL}(2, \mathbf{C})$ with $f$ a primitive elliptic of order $n \geq 3, g$ elliptic of order 2 and $\gamma(f, g) \neq 0, \beta(f)$. Then $G$ is a discrete subgroup of an arithmetic group if

1. $\gamma(f, g)$ is the root of a monic polynomial $p(z, \beta(f))$ in $z$ and $\beta(f)$ with integer coefficients;

2. the roots of $p\left(z, \beta\left(f^{k}\right)\right)$ are real and lie in the interval $\left(\beta\left(f^{k}\right), 0\right)$ for all $k$ such that $(k, n)=1$ and $2 \leq k \leq n / 2$;

3. if $\gamma(f, g)$ and $\bar{\gamma}(f, g)$ are complex, then all other roots of $p(z, \beta(f))$ lie in the interval $(\beta(f), 0)$;

4. if $\gamma(f, g)$ is real, then all other roots of $p(z, \beta(f))$ lie in the interval $(\beta(f), 0)$.

Finally if $n=3,4$ or 6 , then $\beta \in \mathbf{Z}$ and we have the following result.

Theorem 5.14. Let $G=\langle f, g\rangle$ be a subgroup of $\operatorname{PSL}(2, \mathbf{C})$ with $f$ a primitive elliptic of order $n=3,4$ or $6, g$ an elliptic of order 2 and $\gamma(f, g) \neq 0, \beta(f)$. Then $G$ is a discrete subgroup of an arithmetic group if

1. $\gamma(f, g)$ is the root of a monic polynomial $p(z)$ with integer coefficients;

2. if $\gamma(f, g)$ and $\bar{\gamma}(f, g)$ are complex, then all other roots of $p(z)$ lie in the interval $(\beta(f), 0)$;

3. if $\gamma(f, g)$ is real, then all other roots of $p(z)$ lie in the interval $(\beta(f), 0)$.

\section{EXAmples}

In this section we use the discreteness criteria in $\S 5$ to identify a number of interesting two-generator subgroups of arithmetic groups. Some of these were shown in [18], [19] and [20] to be extremal for geometric properties such as minimum axial distance, minimum co-volume and the Margulis constant. A number of these groups are of finite co-volume themselves and are therefore arithmetic; recall that our methods identify the invariant trace field and quaternion algebra.

The groups we consider are generated by a pair of elliptic elements $f$ and $g$ where $f$ is of order $n \geq 3$ and $g$ of order 2. We present these in tabular form for the cases $n=3,4,5,6,7$. By virtue of Theorems 5.13 and 5.14 all of these are subgroups of arithmetic groups, and hence discrete. We discuss in $\S 7$ and $\S 8$ their relations with small volume hyperbolic 3-orbifolds and in $\S 10$ we determine the groups in which the generator $f$ is a simple elliptic.

We begin with a pair of preliminary remarks. Suppose that $(\gamma, \beta,-4)$ are the parameters of a discrete two-generator group $G=\langle f, g\rangle$ with $f$ of order $n$. Then the Lie product $G^{*}$ of $G$ [18] and the conjugations $\bar{G}$ and $\bar{G}^{*}$ of $G$ and $G^{*}$ by reflection in the real axis are discrete two-generator groups with

$$
\operatorname{par}\left(G^{*}\right)=(\beta-\gamma, \beta,-4), \quad \operatorname{par}(\bar{G})=(\bar{\gamma}, \beta,-4), \quad \operatorname{par}\left(\bar{G}^{*}\right)=(\beta-\bar{\gamma}, \beta,-4) .
$$


See, for example, [18]. Moreover, if $G$ is a subgroup of an arithmetic group, then the same is true of $G^{*}, \bar{G}$ and $\bar{G}^{*}$. Hence it is sufficient to consider only those values of $\gamma$ for which

$$
-2 \sin ^{2}(\pi / n)=\beta / 2 \leq \operatorname{Re}(\gamma), \quad 0 \leq \operatorname{Im}(\gamma)
$$

Next if $\gamma \neq 0, \beta$, then by Lemma 2.4 the hyperbolic distance $\delta=\delta(f, g)$ between the axes of the generators $f$ and $g$ of $G$ is given by

$$
\cosh (2 \delta)=\frac{|\gamma-\beta|+|\gamma|}{|\beta|}
$$

In particular, $\delta=0$ if and only if $\langle f, g\rangle$ is elementary. Hence in our case, $\delta$ is a measure of how much $\langle f, g\rangle$ differs from an elementary group. We see from (6.1) that the corresponding axial distances for $G^{*}, \bar{G}$ and $\bar{G}^{*}$ are equal to that for $G$.

We now give for $n=3,4,5,6,7$ tables of groups $G_{n, i}$ generated by elliptics of orders $n$ and 2 together with

1. the approximate values of their commutator parameters $\gamma_{n, i}$,

2. the minimum polynomial $p_{n, i}$ for $\gamma_{n, i}$ over $\mathbf{Q}(\beta)$,

3. the approximate distance $\delta_{n, i}$ between the axes of the generators of $G_{n, i}$.

When $n=3,4,6, p_{n, i}$ coincides with the minimum polynomial $q_{n, i}$ for $\gamma_{n, i}$ over the field $\mathbf{Q}$ considered in the next section.

These groups were identified by the disk covering procedure described in $\S 2$. The resulting diagrams, showing the only possible values for the parameter $\gamma_{n, i}$ for the cases where $n=3,4,5,6$, are given at the end of this paper in $\S 11$. For an account of the calculations on which these diagrams are based, see [21].

We begin with tables for the groups $G_{3, i}$ and $G_{4, i}$.

TABLE 1 . Groups $G_{3, i}$

\begin{tabular}{|c|c|c|c|}
\hline$i$ & $\gamma_{3, i}$ & $p_{3, i}$ & $\delta_{3, i}$ \\
\hline 1 & -1 & $z+1$ & 0 \\
\hline 2 & -.3819 & $z^{2}+3 z+1$ & 0 \\
\hline 3 & $-1.5+.6066 i$ & $z^{4}+6 z^{3}+12 z^{2}+9 z+1$ & .1970 \\
\hline 4 & $-.2118+.4013 i$ & $z^{4}+5 z^{3}+7 z^{2}+3 z+1$ & .2108 \\
\hline 5 & $-.5803+.6062 i$ & $z^{3}+4 z^{2}+4 z+2$ & .2337 \\
\hline 6 & $-1.1225+.7448 i$ & $z^{3}+5 z^{2}+8 z+5$ & .2448 \\
\hline 7 & $-.3376+.5622 i$ & $z^{3}+3 z^{2}+2 z+1$ & .2480 \\
\hline 8 & $-.9236+.8147 i$ & $z^{4}+5 z^{3}+8 z^{2}+6 z+1$ & .2740 \\
\hline 9 & $-1.5+.8660 i$ & $z^{2}+3 z+3$ & .2746 \\
\hline 10 & $-.7672+.7925 i$ & $z^{3}+4 z^{2}+5 z+3$ & .2770 \\
\hline 11 & $.0611+.3882 i$ & $z^{4}+5 z^{3}+6 z^{2}+1$ & .2788 \\
\hline 12 & .2469 & $z^{3}+4 z^{2}+3 z-1$ & .2831 \\
\hline 13 & $-.5284+.7812 i$ & $z^{6}+8 z^{5}+24 z^{4}+35 z^{3}+28 z^{2}+12 z+1$ & .2944 \\
\hline 14 & $-.1153+.5897 i$ & $z^{3}+3 z^{2}+z+1$ & .2970 \\
\hline
\end{tabular}


TABLE 2. Groups $G_{4, i}$

\begin{tabular}{|c|c|c|c|}
\hline$i$ & $\gamma_{4, i}$ & $p_{4, i}$ & $\delta_{4, i}$ \\
\hline 1 & -1 & $z+1$ & 0 \\
\hline 2 & $-.5+.8660 i$ & $z^{2}+z+1$ & .4157 \\
\hline 3 & $-.1225+.7448 i$ & $z^{3}+2 z^{2}+z+1$ & .4269 \\
\hline 4 & $-1+i$ & $z^{2}+2 z+2$ & .4406 \\
\hline 5 & $-.6588+1.1615 i$ & $z^{3}+3 z^{2}+4 z+3$ & .5049 \\
\hline 6 & $.2327+.7925 i$ & $z^{3}+z^{2}+1$ & .5225 \\
\hline 7 & $-.2281+1.1151 i$ & $z^{3}+2 z^{2}+2 z+2$ & .5297 \\
\hline 8 & $.4196+.6062 i$ & $z^{3}+z^{2}-z+1$ & .5297 \\
\hline 9 & $i$ & $z^{2}+1$ & .5306 \\
\hline 10 & .6180 & $z^{2}+z-1$ & .5306 \\
\hline 11 & $-1+1.2720 i$ & $z^{4}+4 z^{3}+7 z^{2}+6 z+1$ & .5306 \\
\hline 12 & $-.4063+1.1961 i$ & $z^{4}+3 z^{3}+4 z^{2}+4 z+1$ & .5345 \\
\hline 13 & $.7881+.4013 i$ & $z^{4}+z^{3}-2 z^{2}+1$ & .6130 \\
\hline
\end{tabular}

Tables 3 and 5 contain the groups for $n=5$ and 7 . Here $p_{n, i}$ is a polynomial in $z$ and $\beta$ to which Theorem 5.13 applies.

TABLE 3 . Groups $G_{5, i}$

\begin{tabular}{|c|c|c|c|}
\hline$i$ & $\gamma_{5, i}$ & $p_{5, i}$ & $\delta_{5, i}$ \\
\hline 1 & -.3819 & $z-\beta-1$ & 0 \\
\hline 2 & $-.6909+.7228 i$ & $z^{2}-\beta z+1$ & .4568 \\
\hline 3 & $.1180+.6066 i$ & $z(z-\beta-1)^{2}-\beta-1$ & .5306 \\
\hline 4 & $-.1909+.9815 i$ & $z^{2}-(\beta+1) z+1$ & .6097 \\
\hline 5 & .6180 & $z-\beta-2$ & .6268 \\
\hline 6 & $.2527+.8507 i$ & $z(z-\beta-1)^{2}+1$ & .6514 \\
\hline 7 & $-.6909+1.2339 i$ & $z^{2}-\beta z+2$ & .6717 \\
\hline 8 & $-.3819+1.2720 i$ & $z^{3}-(2 \beta+1) z^{2}+\left(\beta^{2}+\beta+2\right) z-2 \beta-1$ & .6949 \\
\hline 9 & $.1180+1.1696 i$ & $z^{3}-(2 \beta+2) z^{2}+\left(\beta^{2}+2 \beta+2\right) z-\beta$ & .7195 \\
\hline 10 & $-.0817+1.2880 i$ & $z^{4}-(2 \beta+1) z^{3}+\left(\beta^{2}+\beta+2\right) z^{2}-2 \beta z+1$ & .7273 \\
\hline 11 & $.6180+.7861 i$ & $z^{3}-(2 \beta+3) z^{2}+\left(\beta^{2}+3 \beta+2\right) z+1$ & .7323 \\
\hline 12 & $.8776+.5825 i$ & $z(z-\beta)(z-\beta-2)^{2}+1$ & .7725 \\
\hline
\end{tabular}

TABLE 4 . Groups $G_{6, i}$

\begin{tabular}{|c|c|c|c|}
\hline$i$ & $\gamma_{6, i}$ & $p_{6, i}$ & $\delta_{6, i}$ \\
\hline 1 & $-.5+.8660 i$ & $z^{2}+z+1$ & .6584 \\
\hline 2 & $i$ & $z^{2}+1$ & .7642 \\
\hline 3 & $.5+.8660 i$ & $z^{2}-z+1$ & .8314 \\
\hline 4 & $-.5+1.3228 i$ & $z^{2}+z+2$ & .8500 \\
\hline 5 & $-.2150+1.3071 i$ & $z^{3}+z^{2}+2 z+1$ & .8539 \\
\hline 6 & $.3411+1.1615 i$ & $z^{3}+z+1$ & .8786 \\
\hline 7 & 1 & $z-1$ & .8813 \\
\hline 8 & $.8774+.7448 i$ & $z^{3}-z^{2}+1$ & .9106 \\
\hline
\end{tabular}


TABle 5. Groups $G_{7, i}$

\begin{tabular}{|c|c|c|c|}
\hline$i$ & $\gamma_{7, i}$ & $p_{7, i}$ & $\delta_{7, i}$ \\
\hline 1 & .2469 & $z-\beta-1$ & .5452 \\
\hline 2 & $-.3765+.9264 i$ & $z^{2}-\beta z+1$ & .8162 \\
\hline 3 & 1.2469 & $z-\beta-2$ & 1.0704 \\
\hline
\end{tabular}

We conclude this section with two examples to illustrate how the above tables are constructed. The groups we consider, $G_{3,2}$ and $G_{5,2}$, are those where the minimum distances between the axes of elliptics of orders 3 and 2 and orders 5 and 2 are realized.

In the first example, $\beta=\beta(f)=-3$ and the disk covering procedure shows that the polynomial

$$
z(z+3)\left(z^{2}+3 z+3\right)\left(z^{4}+6 z^{3}+12 z^{2}+9 z+1\right)^{2}\left(z^{4}+6 z^{3}+12 z^{2}+9 z+3\right),
$$

corresponding to the word $h f h^{-1} f h f h^{-1}$ where $h=g f g^{-1} f g f^{-1} g^{-1}$, maps

$$
\gamma_{3,2}=-3 / 2+i \sqrt{(2 \sqrt{5}-3) / 4} \approx-1.5+.606658 i
$$

onto 0. Then

$$
p_{3,2}(z)=z^{4}+6 z^{3}+12 z^{2}+9 z+1
$$

is the minimum polynomial for $\gamma_{3,2}$ and it is easy to check that $p_{3,2}$ has real roots $-.13324,-2.86676$ which lie in the interval $(-3,0)$. Hence we can apply Theorem 5.14 to conclude that $G_{3,2}$ is a subgroup of an arithmetic group and hence discrete.

In the second case,

$$
\beta=\beta(f)=(\sqrt{5}-5) / 2, \quad \beta\left(f^{2}\right)=(-\sqrt{5}-5) / 2
$$

and from the disk covering argument we find that

$$
p_{5,2}(z, \beta)=z^{2}-\beta z-\beta
$$

is the minimum polynomial for

$$
\gamma_{5,2}=(\sqrt{5}-5) / 4+i \sqrt{(5 \sqrt{5}-7) / 8} \approx-.690983+.722871 i
$$

over $\mathbf{Q}(\beta)$. Then $\gamma_{5,2}$ and $\bar{\gamma}_{5,2}$ are the roots of $p_{5,2}(z, \beta(f))$, the roots -.301522 and -3.31651 of $p_{5,2}\left(z, \beta\left(f^{2}\right)\right)$ are both real and the desired conclusion follows from Theorem 5.13.

\section{Small VOlume ARIThmetic ORBIFOldS}

We now discuss the minimal co-volume arithmetic Kleinian groups that the groups $G_{n, i}$ embed in. We deal with the case $n=3$ in some detail and in $\S 8$ merely state the salient information for the others in tabulated form.

We begin with some comments on the more detailed arithmetic structure associated to these groups. For this we need some additional results and terminology, for which we refer the reader to [42].

Let $B$ be a quaternion algebra over a number field $k$. The isomorphism class of $B$ is determined by those places of $k$, both Archimedean and non-Archimedean, at which $B$ is ramified. The number of ramified places is always even. The nonArchimedean places correspond to the prime ideals in $R$, the ring of integers in $k$. 
If $\mathcal{O}$ is an order in $B$, then its discriminant $d(\mathcal{O})$ is an ideal of $R$. Furthermore, $\mathcal{O}$ will be a maximal ideal precisely when $d(\mathcal{O})$ is the product of those ideals at which $B$ is ramified. If $\mathcal{M}$ is an order of $B$ such that $\mathcal{M} \subset \mathcal{O}$, then $d(\mathcal{O}) \mid d(\mathcal{M})$. In the cases where $\mathcal{O}$ is a free $R$-module with basis $\left\{f_{1}, f_{2}, f_{3}, f_{4}\right\}$, the discriminant can be determined from the formula

$$
d(\mathcal{O})^{2}=\left\langle\operatorname{Det}\left(\operatorname{tr}_{B}\left(f_{i} f_{j}\right)\right)\right\rangle
$$

where $\operatorname{tr}_{B}: B \rightarrow k$ is the reduced trace.

The following lemma will prove useful in our calculations.

Lemma 7.1. Let $G$ be a finite co-volume Kleinian group such that $\operatorname{tr}(G)$ consists of algebraic integers and let $R$ denote the ring of integers in $\mathbf{Q}(\operatorname{tr}(G))$. If $\langle f, g\rangle$ is a non-elementary subgroup of $G$, then $R[1, f, g, f g]$ is an order of $A G$.

Proof. Recall that $1, f, g, f g$ span $A G$ over the trace field. Thus since $R[1, f, g, f g]$ contains a basis of $A G$, is finitely generated and contains $R$, it suffices to prove that all products of the basis elements can be expressed as $R$-combinations of the basis elements. There are several obvious ones and these together with the following identities prove the lemma.

$$
\begin{aligned}
f^{2} & =\operatorname{tr}(f) f-1, \\
f^{2} g & =\operatorname{tr}(f) f g-g \\
(f g)^{2} & =\operatorname{tr}(f g) f g-1 \\
f g f & =-\operatorname{tr}(g) 1+\operatorname{tr}(f g) f+g, \\
g f+f g & =(\operatorname{tr}(f g)-\operatorname{tr}(f) \operatorname{tr}(g)) 1+\operatorname{tr}(g) f+\operatorname{tr}(f) g .
\end{aligned}
$$

We adopt some notation that will be used in the next two sections. For $n=3,4,5,6,7$ let $k G_{n, i}$ and $A_{n, i}$ denote the invariant trace field and invariant quaternion algebra of $G_{n, i}$.

We now fix attention on the case of $n=3$ and, in what follows, suppress the subscript 3 for convenience of notation. Next, referring to Table 1 in the cases $i=1,2$ and 12 , since the corresponding commutator parameter is real, one checks easily that these groups are the $(2,3,4)$ spherical triangle group, the $(2,3,5)$ spherical triangle group and the $(2,3,7)$ Fuchsian triangle group. Also in the case $i=13$, the invariant trace field has degree 6 and we will not make any further comment on this case. Thus we concentrate on the cases when the commutator parameter is not real and the relevant polynomials have degree 2,3 and 4 .

Let $\mathcal{O}_{i}$ denote the $R_{k G_{i}}$-submodule of $A_{i}$ generated over $R_{k G_{i}}$ by the elements

$$
\left\{1, g_{i} f_{i} g_{i}^{-1}, f_{i}^{-1}, g_{i} f_{i} g_{i}^{-1} f_{i}^{-1}\right\}
$$

note that since $f_{i}$ has order $3, f_{i}$ and $g_{i} f_{i} g_{i}^{-1}$ lie in $G_{i}^{(2)}$. Lemma 7.1 shows that $\mathcal{O}_{i}$ is an order of $A_{i}$.

By Theorem 5.10, the algebras $A_{i}$ have the following Hilbert symbols,

$$
\left(\frac{-3,\left(\gamma_{i}+3\right) \gamma_{i}}{k G_{i}}\right)
$$

It is an easy calculation using the comments above to compute the discriminant of $\mathcal{O}_{i}$, namely

$$
d\left(\mathcal{O}_{i}\right)=\left\langle\operatorname{tr}\left(\left[f_{i}^{-1}, g_{i} f_{i} g_{i}^{-1}\right]\right)-2\right\rangle=\left\langle\gamma_{i}\left(\gamma_{i}+3\right)\right\rangle .
$$

We first deal with the case of the quartics and cubics.

Lemma 7.2. For each $i \neq 10$, corresponding to a cubic or quartic, $\mathcal{O}_{i}$ is a maximal order. 
Proof. Using the earlier remarks we need to compute the ramification of these quaternion algebras and compare them with $\gamma_{i}\left(\gamma_{i}+3\right)$. Since each ramified prime must divide $d\left(\mathcal{O}_{i}\right)$, it is expeditious to calculate $d\left(\mathcal{O}_{i}\right)$ in the first instance.

In all the quartic cases, a simple calculation shows that $\gamma_{i}\left(\gamma_{i}+3\right)$ is a unit, so that $d\left(\mathcal{O}_{i}\right)=R_{k G_{i}}$, from which it follows that $A_{i}$ cannot have any finite ramification and $\mathcal{O}_{i}$ is maximal.

A variation of this argument works in the cubic cases, $i \neq 10$. For the norm of $\gamma_{i}\left(\gamma_{i}+3\right)$ in each of these cases is a rational prime so that $d\left(\mathcal{O}_{i}\right)$ is a prime ideal $\mathcal{P}_{p_{i}}$. As the cardinality of the set of places ramified in any $A_{i}$ is even and as each $A_{i}$ here is ramified at exactly one real place, $A_{i}$ must be ramified at at least one prime ideal. Thus it is ramified at precisely the one ideal $\mathcal{P}_{p_{i}}$ and $\mathcal{O}_{i}$ is maximal. For later computations, we note that $p_{5}=2, p_{6}=5, p_{7}=5, p_{14}=2$.

For the case $i=10$ we argue as follows. The discriminant of $k G_{10}$ is -31 and by the fact that there is a unique cubic with one complex place and discriminant -31 , an alternative description of $k G_{10}$ is $k G_{10}=\mathbf{Q}(u)$ where $u^{3}+u+1=0$; cf. [36]. The ring of integers in this field has a unique prime ideal $\mathcal{P}_{3}$ of norm 3 and $\mathcal{P}_{3}=\left\langle\gamma_{10}\right\rangle$. Now $\gamma_{10}+3$ also has norm 3 so that $\gamma_{10}\left(\gamma_{10}+3\right)=v \gamma_{10}^{2}$ where $v$ is a unit. Thus $d(\mathcal{O})=\mathcal{P}_{3}^{2}$. As in the above proof $A_{10}$ must be ramified at at least one prime and so must be ramified at precisely the one finite place corresponding to $\mathcal{P}_{3}$. Note that $\mathcal{O}_{10}$ is not a maximal order in this case.

We now compute maximal discrete groups in which the groups $G_{i}$, with $i$ as indicated above, are subgroups. To do this we make use of the description of maximal groups due to Borel [5]. First we consider only cubics and quartics and we assume that $i \neq 10$. The case $i=10$ will be dealt with separately.

Borel's classification of maximal groups in the commensurablity class says that if $\mathcal{O}$ is a maximal order of the quaternion algebra $B$, then the group $P \rho(\operatorname{Norm}(\mathcal{O}))$ (recall the proof of Theorem 5.10) is a maximal group of minimal co-volume in that commensurability class. We denote this maximal group by $\Gamma_{\emptyset, \emptyset}$ in the notation of [5]. Borel actually shows there are infinitely many maximal groups in the commensurability class of an arithmetic group; see the case of $i=10$ below.

As in the proof of Theorem 5.10, since $g_{i}$ normalizes $\left\langle f_{i}, g_{i} f_{i} g_{i}^{-1}\right\rangle$, we deduce that $G_{i}<P \rho\left(\operatorname{Norm}\left(\mathcal{O}_{i}\right)\right)$. By Lemma 7.2 , for $i \neq 10 \mathcal{O}_{i}$ is maximal and we obtain the following result.

Corollary 7.3. For $i \neq 10, G_{i}$ is a subgroup of the group $P \rho\left(\operatorname{Norm}\left(\mathcal{O}_{i}\right)\right)=\Gamma_{\emptyset, \emptyset}^{(i)}$.

For the case $i=10$, we utilize more of Borel's construction of maximal groups referred to above. We briefly recall the relevant points. We recommend the reader have [5] at hand. In what follows $B$ is a quaternion algebra over the number field $k$ with one complex place, which is ramified at all real places. Let $V$ denote the set of all finite places of $k$, and $\operatorname{Ram}_{f}(B)$ denote the finite places which ramify $B$.

Borel proves that to any pair $S$ and $S^{\prime}$ of finite (possibly empty), disjoint subsets of $V \backslash \operatorname{Ram}_{f}(B)$, one can associate a group $\Gamma_{S, S^{\prime}}$. Moreover it is shown in [5] that any arithmetic Kleinian group $\Gamma$ in the commensurability class determined by $B$ is conjugate to a subgroup of some $\Gamma_{S, S^{\prime}}$. We will not make use of this explicitly here.

Let $\nu \in V \backslash \operatorname{Ram}_{f}(B)$ and denote by $\mathcal{C}_{\nu}$ the Bruhat-Tits Building of $\mathrm{SL}\left(2, k_{\nu}\right)$, which in these cases is a tree. The vertices of this tree are maximal orders of $M\left(2, k_{\nu}\right)$, and the $\mathrm{SL}\left(2, k_{\nu}\right)$ action on the vertices forms 2 orbits. The groups $\Gamma_{S, S^{\prime}}$ 
are described in terms of vertex and edge stabilizers of these trees. Since $B$ is unramified at $\nu$ we get $B_{\nu}=B \otimes_{k} k_{\nu} \cong M\left(2, k_{\nu}\right)$. Thus for any group in the commensurability class determined by $B$ we have an induced action on $\mathcal{C}_{\nu}$.

In our case, since $d\left(\mathcal{O}_{10}\right)$ is divisible only by $\mathcal{P}_{3}$, if $\nu \in V \backslash \mathcal{P}_{3}$, then $\mathcal{O}_{10}$, localized at $\nu$ is maximal; see [42]. Given any element of $\operatorname{Norm}\left(\mathcal{O}_{10}\right)$, the image of this in $A_{10, \nu}$ stabilizes the vertex of $\mathcal{C}_{\nu}$ associated to $\mathcal{O}_{10}$ localized at $\nu$. It now follows from this and Borel's description of maximal groups that $P \rho\left(\operatorname{Norm}\left(\mathcal{O}_{10}\right)\right) \subset \Gamma_{\emptyset, \emptyset}^{10}$; see section 4 of [5]. Hence we can conclude that Corollary 7.3 also holds for $i=10$.

To compute the minimal co-volumes in the cubic and quartic cases we use the formula of [5].

Quartic cases. We have seen from the proof of Lemma 7.2 that in the case of $i=3$, 4, 8 and 11 the quaternion algebras $A_{i}$ are unramified at all finite places. It is an easy calculation to show that the class number of these quartic fields is 1 and further that the structure of the group of units of these fields implies that the co-volume of the groups $\Gamma_{\emptyset, \emptyset}^{(i)}$ is

$$
\frac{\left|d_{k G_{i}}\right|^{3 / 2} \zeta_{k G_{i}}(2)}{2^{7} \pi^{6}}
$$

where $d_{k G_{i}}$ is the discriminant and $\zeta_{k G_{i}}(s)$ is the Dedekind zeta function of $k G_{i}$; cf. [5]. Reasonably good estimates for $\zeta_{k G_{i}}(2)$ are obtained by considering primes of small norm in $k G_{i}$ in the first few terms of the Euler product expansion of $\zeta_{k G_{i}}(2)$.

1. The polynomial $z^{4}+6 z^{3}+12 z^{2}+9 z+1$ has discriminant -275 , and this is the smallest discriminant of a field with one complex place; cf. [26]. As shown in [9] for example this is the invariant quaternion algebra of the smallest volume orientable arithmetic hyperbolic 3-orbifold. This minimal volume is approximately $0.03905 \ldots$

2. The polynomial $z^{4}+5 z^{3}+7 z^{2}+3 z+1$ has discriminant -283 and hence the discriminant of $k G_{4}$ coincides with this. There is a unique quartic field of this discriminant; cf. [26]. The minimal co-volume in the commensurability class determined by $A_{4}$ is approximately 0.0408 , the second smallest volume known. This orbifold is commensurable with $(5,1)$-Dehn surgery on the figure eight knot complement [8].

3. The other two quartics have discriminants -491 and -563 , respectively. By comparing with [26], these are the discriminants of the invariant trace-fields. The smallest co-volume in the commensurability classes are approximately $0.1028 \ldots$ and $0.1274 \ldots$, respectively.

Cubic cases. The corresponding volume formula in this case is

$$
\frac{\left|d_{k G_{i}}\right|^{3 / 2} \zeta_{k G_{i}}(2)(N \mathcal{P}-1)}{2^{6} \pi^{4}}
$$

using the same terminology as above. The additional term $N \mathcal{P}$ denotes the norm of the prime ideal $\mathcal{P}$ ramifying the algebra in each case.

1. The polynomial $z^{3}+5 z^{2}+8 z+5$ has discriminant -23 which is necessarily the discriminant of the invariant trace-field. The minimal co-volume of a group in this commensurability class is approximately 0.07859 . This orbifold is commensurable with the Weeks' manifold of volume 0.942707 . .

There is a unique field of discriminant -23 . Hence a non-real root of the polynomial $z^{3}+3 z^{2}+2 z+1$ also generates $k G_{6}$. In fact, up to conjugation, $G_{7}$ is a subgroup of the same maximal group as contains $G_{6}$. 
2. The polynomial $z^{3}+4 z^{2}+5 z+3$ has discriminant -31 which also must coincide with the discriminant of $k G_{10}$. Following the proof of Lemma 7.2, we observed that $A_{10}$ is ramified at the place $\nu_{3}$ corresponding to $\mathcal{P}_{3}$. From our comments above $G_{10}$ is a subgroup of the minimal co-volume group which in this case is approximately 0.06596 .

(In unpublished calculations we have shown that this orbifold is commensurable with $(8,1)$-Dehn surgery on the figure-eight knot complement, and contains the orbifold group of $(3,0),(3,0)$-Dehn surgery on the Whitehead link as a subgroup of index 8 . See also [27].)

3. The polynomial $z^{3}+4 z^{2}+4 z+2$ has discriminant -44 , which coincides with the discriminant of $k G_{5}$. The smallest co-volume of a group in the commensurability class is approximately 0.066194 .

4. The polynomial $z^{3}+3 z^{2}+z+1$ has discriminant -76 which is the discriminant of $k G_{14}$. The smallest co-volume in this commensurability class is 0.1642 .

This leaves the case of $i=9$ for which the relevant polynomial is quadratic. A root of this polynomial generates the field $\mathbf{Q}(\sqrt{-3})$. Moreover notice that directly from the polynomial we can read off that $z^{2}+3 z=-3$. Hence the algebra is

$$
\left(\frac{-3,-3}{\mathbf{Q}(\sqrt{-3})}\right) \text {. }
$$

By [42], this is isomorphic to $M(2, \mathbf{Q}(\sqrt{-3}))$. Indeed in this case, the trace-field of $G_{9}$ is $\mathbf{Q}(\sqrt{-3})$ and therefore one easily deduces from [35] for example that $G_{9}$ is conjugate to a subgroup of $\operatorname{PSL}\left(2, \mathrm{O}_{3}\right)$ and hence of the minimal co-volume group $\operatorname{PGL}\left(2, O_{3}\right)$. This group has co-volume $0.08457 \ldots$ and is the smallest volume of an orientable cusped hyperbolic 3 -orbifold [34].

\section{Axial distance and small volumes}

In this section, we summarize calculations similar to those carried out in $\S 7$ for the cases where $n=4,5,6,7$, omitting those where the degree of the minimum polynomial exceeds four. There are some additional complications which we comment on below, but we make no attempt at detailing all the calculations.

In each case, the Hilbert symbol of the invariant quaternion algebra is easily computed in terms of the parameter $\gamma_{n, i}$ as

$$
\left(\frac{-4 \sin ^{2}(2 \pi / n), \gamma_{n, i}\left(\gamma_{n, i}+4 \sin ^{2}(\pi / n)\right)}{k G_{n, i}}\right) .
$$

As in the case $n=3$, we construct from the two-generator group $\langle f, g\rangle$ with $f^{n}=g^{2}=1$ a suitable order which enables us to determine the finite ramification of the quaternion algebra and a small co-volume group, containing the normalizer of the order, in which the group $G_{n, i}$ lies.

Now for $n=3,4,5,6,7$ we give tables for the groups $G_{n, i}$ of $\S 6$ with

1. the minimum polynomial $q_{n, i}$ over $\mathbf{Q}$ for the commutator parameter $\gamma_{n, i}$ of $G_{n, i}$

2. the discriminant $d_{G_{n, i}}$ of the trace-field $k G_{n, i}$,

3. the finite places $\operatorname{Ram}_{f}$ where the invariant quaternion algebra ramifies,

4. the approximate distance $\delta_{n, i}$ between the axes of the generators of $G_{n, i}$,

5. the approximate smallest co-volume $V_{n, i}$ of an arithmetic Kleinian group in which we know $G_{n, i}$ can be embedded. 
TABLE 6 . Co-volume of group containing $G_{3, i}$

\begin{tabular}{|c|c|c|c|c|c|}
\hline$i$ & $q_{3, i}$ & $d_{k G_{3, i}}$ & $\operatorname{Ram}_{f}$ & $\delta_{3, i}$ & $V_{3, i}$ \\
\hline 1 & $z+1$ & -- & -- & 0 & $S_{4}$ \\
\hline 2 & $z^{2}+3 z+1$ & -- & -- & 0 & $A_{5}$ \\
\hline 3 & $z^{4}+6 z^{3}+12 z^{2}+9 z+1$ & -275 & $\emptyset$ & .1970 & .0390 \\
\hline 4 & $z^{4}+5 z^{3}+7 z^{2}+3 z+1$ & -283 & $\emptyset$ & .2108 & .0408 \\
\hline 5 & $z^{3}+4 z^{2}+4 z+2$ & -44 & $\mathcal{P}_{2}$ & .2337 & .0661 \\
\hline 6 & $z^{3}+5 z^{2}+8 z+5$ & -23 & $\mathcal{P}_{5}$ & .2448 & .0785 \\
\hline 7 & $z^{3}+3 z^{2}+2 z+1$ & -23 & $\mathcal{P}_{5}$ & .2480 & .0785 \\
\hline 8 & $z^{4}+5 z^{3}+8 z^{2}+6 z+1$ & -563 & $\emptyset$ & .2740 & .1274 \\
\hline 9 & $z^{2}+3 z+3$ & -3 & $\emptyset$ & .2746 & .0845 \\
\hline 10 & $z^{3}+4 z^{2}+5 z+3$ & -31 & $\mathcal{P}_{3}$ & .2770 & .0659 \\
\hline 11 & $z^{4}+5 z^{3}+6 z^{2}+1$ & -491 & $\emptyset$ & .2788 & .1028 \\
\hline 12 & $z^{3}+4 z^{2}+3 z-1$ & -- & -- & .2831 & Fuch. \\
\hline 13 & $z^{6}+8 z^{5}+24 z^{4}+35 z^{3}+28 z^{2}+12 z+1$ & $?$ & $?$ & .2944 & $?$ \\
\hline 14 & $z^{3}+3 z^{2}+z+1$ & -76 & $\mathcal{P}_{2}$ & .2970 & .1654 \\
\hline
\end{tabular}

TABLE 7. Co-volume of group containing $G_{4, i}$

\begin{tabular}{|c|c|c|c|c|c|}
\hline$i$ & $q_{4, i}$ & $d_{k G_{4, i}}$ & $\operatorname{Ram}_{f}$ & $\delta_{4, i}$ & $V_{4, i}$ \\
\hline 1 & $z+1$ & -- & -- & 0 & $S_{4}$ \\
\hline 2 & $z^{2}+z+1$ & -3 & $\left\{\mathcal{P}_{4}, \mathcal{P}_{3}\right\}$ & .4157 & .1268 \\
\hline 3 & $z^{3}+2 z^{2}+z+1$ & -23 & $\mathcal{P}_{8}$ & .4269 & .1374 \\
\hline 4 & $z^{2}+2 z+2$ & -4 & $\emptyset$ & .4406 & .2289 \\
\hline 5 & $z^{3}+3 z^{2}+4 z+3$ & -31 & $\mathcal{P}_{3}$ & .5049 & .2968 \\
\hline 6 & $z^{3}+z^{2}+1$ & -31 & $\mathcal{P}_{3}$ & .5225 & .2968 \\
\hline 7 & $z^{3}+2 z^{2}+2 z+2$ & -44 & $\mathcal{P}_{2}$ & .5297 & .0661 \\
\hline 8 & $z^{3}+z^{2}-z+1$ & -44 & $\mathcal{P}_{2}$ & .5297 & .0661 \\
\hline 9 & $z^{2}+1$ & -4 & $\emptyset$ & .5306 & .1526 \\
\hline 10 & $z^{2}+z-1$ & -- & -- & .5306 & Fuch. \\
\hline 11 & $z^{4}+4 z^{3}+7 z^{2}+6 z+1$ & -400 & $\emptyset$ & .5306 & .0717 \\
\hline 12 & $z^{4}+3 z^{3}+4 z^{2}+4 z+1$ & -331 & $\emptyset$ & .5345 & .4475 \\
\hline 13 & $z^{4}+z^{3}-2 z^{2}+1$ & -283 & $\emptyset$ & .6130 & .3475 \\
\hline
\end{tabular}

TABle 8. Co-volume of group containing $G_{5, i}$

\begin{tabular}{|c|c|c|c|c|c|}
\hline$i$ & $q_{5, i}$ & $d_{k G_{5, i}}$ & $\operatorname{Ram}_{f}$ & $\delta_{5, i}$ & $V_{5, i}$ \\
\hline 1 & $z^{2}+3 z+1$ & -- & -- & 0 & $A_{5}$ \\
\hline 2 & $z^{4}+5 z^{3}+7 z^{2}+5 z+1$ & -475 & $\emptyset$ & .4568 & .0933 \\
\hline 3 & $z^{4}+4 z^{3}+2 z^{2}+z+1$ & -275 & $\emptyset$ & .5306 & .0390 \\
\hline 4 & $z^{4}+3 z^{3}+3 z^{2}+3 z+1$ & -275 & $\emptyset$ & .6097 & .0390 \\
\hline 5 & $z^{2}+z-1$ & -- & -- & .6268 & Fuch. \\
\hline 6 & $z^{6}+6 z^{5}+11 z^{4}+8 z^{3}+7 z^{2}+7 z+1$ & $?$ & $?$ & .6514 & $?$ \\
\hline 7 & $z^{4}+5 z^{3}+9 z^{2}+10 z+4$ & -775 & $\left\{\mathcal{P}_{4}, \mathcal{P}_{4}^{\prime}\right\}$ & .6717 & .461 \\
\hline 8 & $z^{4}+6 z^{3}+12 z^{2}+14 z+11$ & -400 & $\emptyset$ & .6949 & .0717 \\
\hline 9 & $z^{4}+4 z^{3}+4 z^{2}+5 z+5$ & -475 & $\emptyset$ & .7195 & .0933 \\
\hline 10 & $z^{6}+6 z^{5}+12 z^{4}+16 z^{3}+17 z^{2}+8 z+1$ & $?$ & $?$ & .7273 & $?$ \\
\hline 11 & $z^{4}+2 z^{3}-2 z^{2}+2 z+1$ & -400 & $\emptyset$ & .7323 & .0717 \\
\hline 12 & $z^{6}+5 z^{5}+3 z^{4}-8 z^{3}+z^{2}+8 z+1$ & $?$ & $?$ & .7725 & $?$ \\
\hline
\end{tabular}


TABLE 9. Co-volume of group containing $G_{6, i}$

\begin{tabular}{|c|c|c|c|c|c|}
\hline$i$ & $q_{6, i}$ & $d_{k G_{6, i}}$ & $\operatorname{Ram}_{f}$ & $\delta_{6, i}$ & $V_{6, i}$ \\
\hline 1 & $z^{2}+z+1$ & -3 & $\emptyset$ & .6584 & .0845 \\
\hline 2 & $z^{2}+1$ & -4 & $\left\{\mathcal{P}_{9}, \mathcal{P}_{2}\right\}$ & .7642 & .3053 \\
\hline 3 & $z^{2}-z+1$ & -3 & $\emptyset$ & .8314 & .1691 \\
\hline 4 & $z^{2}+z+2$ & -7 & $\left\{\mathcal{P}_{2}, \mathcal{P}_{2}^{\prime}\right\}$ & .8500 & .5555 \\
\hline 5 & $z^{3}+z^{2}+2 z+1$ & -23 & $\mathcal{P}_{27}$ & .8539 & .5106 \\
\hline 6 & $z^{3}+z+1$ & -31 & $\mathcal{P}_{3}$ & .8786 & .3298 \\
\hline 7 & $z-1$ & -- & -- & .8813 & Fuch. \\
\hline 8 & $z^{3}-z^{2}+1$ & -23 & $\mathcal{P}_{27}$ & .9106 & .5106 \\
\hline
\end{tabular}

TABle 10. Co-volume of group containing $G_{7, i}$

\begin{tabular}{|c|c|c|c|c|c|}
\hline$i$ & $q_{7, i}$ & $d_{k G_{7, i}}$ & $\operatorname{Ram}_{f}$ & $\delta_{7, i}$ & $V_{7, i}$ \\
\hline 1 & $z^{3}+4 z^{2}+3 z-1$ & -- & -- & .5452 & Fuch. \\
\hline 2 & $z^{6}+7 z^{5}+17 z^{4}+21 z^{3}+17 z^{2}+7 z+1$ & $?$ & $?$ & .8162 & $?$ \\
\hline 3 & $z^{3}+z^{2}-2 z-1$ & -- & -- & 1.0704 & Fuch. \\
\hline
\end{tabular}

Notes.

1. In these tables, we have adopted the notation of labeling a prime ideal of norm $p^{n}$ by $\mathcal{P}_{p^{n}}$. In the cases considered, this uniquely determines the ideal except for the indicated cases where $\mathcal{P}_{2}, \mathcal{P}_{2}^{\prime}$ or $\mathcal{P}_{4}, \mathcal{P}_{4}^{\prime}$ both appear.

2. We have not dealt with the cases where $[\mathbf{Q}(\gamma): \mathbf{Q}]>4$.

\section{Remarks.}

1. As remarked in $\S 7$, any subgroup of an arithmetic Kleinian group is contained in a group $\Gamma_{S, S^{\prime}}[5]$. In all cases when $n=3$, applying this to $G_{3, i}$ we have $S=\phi$. That is not the situation in many cases when $n=4,5,6$, but in most cases $S$ consists of a single prime ideal. The volume of $\Gamma_{S, S^{\prime}}$ is readily calculated from the formulas given in [5].

2. A detailed knowledge of subgroups commensurable with tetrahedral groups enable some groups to be identified immediately by their commutator parameter and so the minimum volume of a group in which they lie is quickly determined. (See comments after the remarks on the tables.) The arithmetic nature of tetrahedral groups is discussed in [31] and [37] from which much of the algebraic data of Table 8 is taken.

3. Table $\mathbf{7}, \mathbf{n}=\mathbf{4}$. The order we have considered is (suppressing subscripts)

$$
R_{k G}\left[1, f^{2},(g f)^{2}, f^{2}(g f)^{2}\right]
$$

which has discriminant $\langle 2 \gamma(\gamma+2)\rangle$. Only in the cases $i=2,3$ is this order maximal. In these cases, the group $G_{4, i}$ is contained in a $\Gamma_{\phi, \phi}$ as is also the case for $i=7,8$ following an argument similar to that used in the case of $G_{3,10}$ in $\S 7$. In the cases $i=4,5,6,12,13, S$ consists of a single prime, while in the case $i=9, S$ may consist of two primes. The groups $G_{4,1}$ and $G_{4,11}$ are subgroups of groups commensurable with tetrahedral groups. 
4. Table $\mathbf{8}, \mathbf{n}=\mathbf{5}$. Referring to Table 3 , note that the polynomials $p_{5, i}$ admit $z+1$ as a factor in the cases $i=3,8,9,10,11,12$. Using this, the field discriminants of Table 8 are readily determined.

The order here is chosen to be

$$
R_{k G}\left[1, g f g^{-1}, f^{-1},[g, f]\right]
$$

which has discriminant $\langle\gamma(\gamma-\beta)\rangle$ where $\beta=(-5+\sqrt{ } 5) / 2$. From this, as before, we determine the finite ramification of the algebra, which here, is only non-empty in the case $i=7$. All other groups arise in considering cocompact tetrahedral groups.

5. Table $\mathbf{9}, \mathbf{n}=\mathbf{6}$. Now we consider the order

$$
R_{k G}\left[1, f^{2}, g f^{-2} g^{-1}, f^{2} g f^{-2} g^{-1}\right]
$$

which has discriminant $\langle 9 \gamma(\gamma+1)\rangle$. For $i=2,5,8$, the order is maximal so that $S=\phi$, while when $i=3,4,6, S$ consists of at most one prime ideal. The group $G_{6,1}$ is commensurable with a tetrahedral group. See also [17].

We now comment on the relationship between some of these groups and tetrahedral groups. Recall by a tetrahedral group we mean the orientation-preserving subgroup of index 2 in the group generated by reflections in the faces of tetrahedron in $\mathbf{H}^{3}$, where some vertices may be ideal. Justifications for the comments below can be deduced for example from [37] where, as here, the notation for the tetrahedra is that of $[6]$.

The groups PGL $(2, \mathbf{Z}[i])$ and $\mathrm{PGL}\left(2, \mathrm{O}_{3}\right)$ are tetrahedral, the tetrahedrons being $T[3,2,2 ; 4,2,4]$ and $T[3,2,2 ; 6,2,3]$. In the tables these arise in the cases of $G_{4,9}$ and PGL $\left(2, O_{3}\right)$ occurs in the cases $G_{3,9}$ and $G_{6,1}$. Other non-cocompact groups which arise although not tetrahedral, contain a tetrahedral group of index 2 . The case $G_{4,4}$ yields a group containing the tetrahedral group $T[4,2,2 ; 4,2,4]$ of index 2 , and the case $G_{6,3}$ yields a group containing the tetrahedral group $T[2,2,3 ; 2,6,3]$ of index 2 .

In the case of order 5 the algebras defined over the quartic fields listed in Table 8 except $i=7$ yield groups commensurable with cocompact tetrahedral groups. In the case where the discriminant is -400 the tetrahedral group is $T[2,2,4 ; 2,3,5]$ (which also arises in the case $G_{4,11}$ ), the discriminants -275 and -475 yield groups commensurable with the tetrahedral groups $T[2,2,3 ; 2,5,3]$ and $T[2,2,5 ; 2,3,5]$ respectively. The case $T[2,2,3 ; 2,5,3]$ also arises in the case $G_{3,3}$.

Finally the order 6 case $G_{6,4}$ yields a group commensurable with the tetrahedral group $T[2,3,4 ; 2,3,4]$.

Recently K. N. Jones and the fourth author have developed a computer program to study explicitly how the geometry and topology of certain arithmetic Kleinian groups varies as the number theoretic data is varied. Among other things it constructs a fundamental polyhedron for the action of certain unit groups of orders and their normalizers in $\mathbf{H}^{3}$, it determines the co-volume and it computes a presentation. We present in Table 11 the results produced by that program for the two-generator groups discussed in this paper. Since all but $G_{4,11}, G_{5,7}, G_{6,4}$ and the Fuchsian examples turn out to have finite co-volume, we conclude that, with these exceptions, all these groups are themselves arithmetic.

Theorem 8.2. The following two-generator Kleinian groups $G_{n, i}$ generated by elliptics of order $n$ and 2 are arithmetic. 
1. $G_{3, i}, i=3, \ldots, 14, i \neq 12,13$,

2. $G_{4, i}, i=2, \ldots, 13, i \neq 10,11$,

3. $G_{5, i}, i=2, \ldots, 11, i \neq 5,6,7,10$,

4. $G_{6, i}, i=1, \ldots, 8, i \neq 4$.

We conclude this section with some philosophical remarks. Several of the algebras and small volume groups arise in the considerations of [10]. This work identifies the smallest volume of an orientable arithmetic hyperbolic 3-manifold, namely that of the Weeks manifold. The content of this article together with [9] and the works [16]-[23] seem to indicate a convergence of arithmetic and geometric ideas. In particular the smallest volume orientable arithmetic orbifold arises as the quotient of hyperbolic 3 -space $\mathbf{H}^{3}$ by a $\mathbf{Z}_{2}$-extension of the tetrahedral group $T[2,3,3 ; 2,5,3]$ (cf. [9]) and it would seem that this article and [16]-[21] are "converging on" the arithmetic orbifold just described as the overall smallest one, as is conjectured.

TABle 11. Co-volumes of $\mathbf{G}_{n, i}$

\begin{tabular}{|c|c|c|c|c|}
\hline$i$ & $G_{3, i}$ & $G_{4, i}$ & $G_{5, i}$ & $G_{6, i}$ \\
\hline 1 & $S_{4}$ & $S_{4}$ & $A_{5}$ & .2537 \\
\hline 2 & $A_{4}$ & .2537 & .0933 & .6106 \\
\hline 3 & .0390 & .1374 & .0390 & .5074 \\
\hline 4 & .0408 & .4579 & .4686 & $\infty$ \\
\hline 5 & .1323 & .5936 & Fuch. & 1.0212 \\
\hline 6 & .1571 & .5936 & $?$ & 1.3193 \\
\hline 7 & .1571 & .7943 & $\infty$ & Fuch. \\
\hline 8 & .1274 & .2647 & .8612 & 1.0212 \\
\hline 9 & .3383 & .9159 & 1.1199 & -- \\
\hline 10 & .2638 & Fuch. & $?$ & -- \\
\hline 11 & .2056 & $\infty$ & .8612 & -- \\
\hline 12 & Fuch. & .8951 & $?$ & -- \\
\hline 13 & $?$ & .3475 & -- & -- \\
\hline 14 & .3308 & -- & -- & -- \\
\hline
\end{tabular}

\section{CRiteria For Simple AXeS}

Suppose that $f$ is an elliptic element of order $n$ in a Kleinian group $G$. Then $f$ is simple if axis $(f)$ is precisely invariant, that is, if for each $h \in G$

$$
h(\operatorname{axis}(f))=\operatorname{axis}\left(h f h^{-1}\right)
$$

either coincides with or is disjoint from $\operatorname{axis}(f)$. If $f$ is not simple, then one of the following is true [4] and [33].

1. The axes of $f$ and $h f h^{-1}$ intersect in $\mathbf{H}^{3}$ and $G$ contains a subgroup isomorphic to the regular solid group $A_{4}, S_{4}$ or $A_{5}$.

2. The axes of $f$ and $h f h^{-1}$ have one endpoint in common and $G$ contains a subgroup isomorphic to the $(3,3,3),(2,4,4)$ or $(2,3,6)$ euclidean triangle group.

We show here that if $G$ is a Kleinian group of finite co-volume, then each of these conditions forces the invariant quaternion algebra of $G$ to satisfy certain criteria. Hence if the algebra fails to satisfy these criteria, then every elliptic in $G$ of order $n \geq 3$ must be simple. 
Case where axes of $\mathbf{f}$ and $\mathbf{h f h} \mathbf{h}^{-1}$ intersect in $\mathbf{H}^{3}$. Let $\mathcal{H}$ denote Hamilton's quaternions so that $\mathcal{H}=\left(\frac{-1,-1}{\mathbf{R}}\right)$ and let $\sigma$ denote the embedding $\sigma: \mathcal{H}^{1} \rightarrow \operatorname{SL}(2, \mathbf{C})$ given by

$$
\sigma\left(a_{0}+a_{1} i+a_{2} j+a_{3} i j\right)=\left(\begin{array}{cc}
a_{0}+a_{1} i & a_{2}+a_{3} i \\
-a_{2}+a_{3} i & a_{0}-a_{1} i
\end{array}\right),
$$

where $\mathcal{H}^{1}$ is the group of elements of norm 1 . If $n$ denotes the norm on $\mathcal{H}$, then there is an epimorphism

$$
\Phi: \mathcal{H}^{1} \rightarrow \mathrm{SO}(3, \mathbf{R})
$$

where $\mathrm{SO}(3, \mathbf{R})$ is represented as the orthogonal group of the quadratic subspace $V$ of $\mathcal{H}$ spanned by $\{i, j, i j\}$, that is, the pure quaternions, equipped with the restriction of the norm form, so that $n\left(x_{1} i+x_{2} j+x_{3} i j\right)=x_{1}^{2}+x_{2}^{2}+x_{3}^{2}$. The mapping $\Phi$ is defined by $\Phi(\alpha)=\phi_{\alpha}$, where

$$
\phi_{\alpha}(\beta)=\alpha \beta \alpha^{-1}, \quad \alpha \in \mathcal{H}^{1}, \beta \in V .
$$

The kernel of $\Phi$ is $\{ \pm 1\}$. The binary tetrahedral group is a central extension of an element of order 2 by the tetrahedral group and can be faithfully represented in $\mathcal{H}$. This is also true for the binary octahedral group and the binary icosahedral group as will now be shown.

If the tetrahedron has its vertices at

$$
i+j+i j, \quad i-j-i j, \quad-i+j-i j, \quad-i-j+i j,
$$

then $\phi_{\alpha_{1}}$ is a rotation of order 2 about the axis through the edge mid-point $i$ if $\alpha_{1}=i$, while $\phi_{\alpha_{2}}$ is a rotation of order 3 about the axis through the vertex $i+j+i j$ when $\alpha_{2}=(1+i+j+i j) / 2$. The binary tetrahedral group $\Gamma_{1}$ is thus generated by $\alpha_{0}=-1, \alpha_{1}, \alpha_{2}$ in $\mathcal{H}^{1}$.

If the cube has its vertices at

$$
\pm i \pm j \pm i j
$$

then $\phi_{\alpha_{3}}$ is a rotation of order 4 about the axis through the mid-point $i$ of a face when $\alpha_{3}=(1+i) / \sqrt{2}$. Thus the binary octahedral group $\Gamma_{2}$ is generated by $\alpha_{0}, \alpha_{2}, \alpha_{3}$.

If the regular icosahedron has its vertices at

$$
\pm i \pm j \pm i j, \quad \pm \tau i \pm \tau^{-1} j, \quad \pm \tau j \pm \tau^{-1} i j, \quad \pm \tau i j \pm \tau^{-1} i,
$$

then $\phi_{\alpha_{4}}$ is a rotation of order 5 about the axis through the mid-point of the face with vertices

$$
i+i+i j, \quad i+j-i j, \quad \tau i+\tau^{-1} j, \quad \tau j+\tau^{-1} i j, \quad \tau j-\tau^{-1} i j,
$$

where $\alpha_{4}=\left(\tau+\tau^{-1} i+j\right) / 2$ and $\tau=(\sqrt{5}+1) / 2$. The binary icosahedral group $\Gamma_{3}$ is then generated by $\alpha_{0}, \alpha_{2}, \alpha_{4}$.

The groups $P \sigma\left(\Gamma_{1}\right) \cong A_{4}, P \sigma\left(\Gamma_{2}\right) \cong S_{4}, P \sigma\left(\Gamma_{3}\right) \cong A_{5}$, where $P$ is the projection $P: \mathrm{SL}(2, \mathbf{C}) \rightarrow \operatorname{PSL}(2, \mathbf{C})$, are said to be in standard form in $\operatorname{PSL}(2, \mathbf{C})$. If $G$ is a discrete group which contains a finite subgroup $F$ isomorphic to one of these regular solid groups, then $G$ can be conjugated so that $F$ is in standard form. Note that if $G$ contains any of these finite groups, it will contain a subgroup isomorphic to $A_{4}$. 
Lemma 9.1. Let $G$ be a Kleinian group of finite co-volume with invariant quaternion algebra $A$ and number field $k$. If $G$ contains a subgroup isomorphic to $A_{4}$, then

$$
A \cong\left(\frac{-1,-1}{k}\right)
$$

In particular, the only finite primes at which $A$ can be ramified are the dyadic primes.

Proof. Suppose that $G$ contains a subgroup isomorphic to $A_{4}$. Since $A_{4}$ has no subgroup of index 2 and $A_{5}$ is simple, we have $A_{n}=A_{n}^{(2)}$ for $n=4$ or 5 . So if $A_{n} \subset G$, then $A_{n} \subset G^{(2)}$. Thus by conjugation, we can assume that $\sigma\left(\Gamma_{1}\right) \subset \mathcal{G}$ where $P \mathcal{G}=G^{(2)}$. Now

$$
A=\left\{\sum a_{i} g_{i}: a_{i} \in k, \quad g_{i} \in \mathcal{G}\right\}
$$

Let $A_{0}=\left(\frac{-1,-1}{\mathbf{Q}}\right)$. Then

$$
A_{0} \cong\left\{\sum a_{i} g_{i}: a_{i} \in \mathbf{Q} \quad, g_{i} \in \sigma\left(\Gamma_{1}\right)\right\}
$$

since $1, i, j, i j \in \Gamma_{1}$. Now the quaternion algebra

$$
\left\{\sum a_{i} g_{i}: a_{i} \in k \quad g_{i} \in \sigma\left(\Gamma_{1}\right)\right\}
$$

lies in $A$, is isomorphic to $A_{0} \otimes_{\mathbf{Q}} k$ and is 4-dimensional. Thus

$$
A \cong\left(\frac{-1,-1}{k}\right)
$$

Finally it follows from this form of the Hilbert symbol, that $A$ splits over all $\mathcal{P}$-adic fields $k_{\mathcal{P}}$, with $\mathcal{P}$ non-dyadic, that is, primes not dividing 2 .

Lemma 9.3. Let $G$ be as in Lemma 9.1. If $G$ contains a subgroup isomorphic to $A_{5}$ and if $[k: \mathbf{Q}]=4$, then $A$ has no finite ramification.

Proof. $A$ can at worst have dyadic finite ramification. Note that, since $G$ must contain an element of order $5, \mathbf{Q}(\sqrt{5}) \subset k$. There is a unique prime $\mathcal{P}$ in $\mathbf{Q}(\sqrt{5})$ such that $\mathcal{P} \mid 2$. So if $\mathcal{P}$ ramifies or is inert in $k \mid \mathbf{Q}(\sqrt{5})$, then there will only be one dyadic prime in $k$ at which $A$ cannot be ramified for parity reasons. Suppose then that $\mathcal{P}$ splits as $\mathcal{P}_{1} \mathcal{P}_{2}$ so that $k_{\mathcal{P}_{1}} \cong k_{\mathcal{P}_{2}} \cong \mathbf{Q}(\sqrt{5})_{\mathcal{P}}$. But, again for parity reasons, the quaternion algebra

$$
\left(\frac{-1,-1}{\mathbf{Q}(\sqrt{5})}\right)
$$

splits in the field $\mathbf{Q}(\sqrt{5})_{\mathcal{P}}$. Hence

$$
\left(\frac{-1,-1}{k}\right)
$$

splits in $k_{\mathcal{P}_{1}}$ and $k_{\mathcal{P}_{2}}$ and $A$ has no finite ramification.

Lemma 9.1 has the following partial converse.

Lemma 9.4. Suppose that $k$ has exactly one complex place. If

$$
A \cong\left(\frac{-1,-1}{k}\right)
$$


then there is an arithmetic Kleinian group in the commensurability class defined by $A$ which contains $S_{4}$. Furthermore if $\mathbf{Q}(\sqrt{5}) \subset k$, then there is a group in the commensurability class containing $A_{5}$.

Proof. To exhibit a group containing $S_{4}$ we proceed as follows. Let $R_{k}$ denote the ring of integers of $k$ and let

$$
\mathcal{O}=R_{k}[1, i, j,(1+i+j+i j) / 2]
$$

It is easily checked that $\mathcal{O}$ is an order in $A$. Note that $\Gamma_{1} \subset \mathcal{O}^{1}$. Furthermore the element $1+i \in \mathcal{O}$ normalizes $\mathcal{O}^{1}$. Thus if $\rho$ is a representation of $A$ into $M(2, \mathbf{C})$, then

$$
P \rho\left(\mathcal{O}^{1}\right) \subset\left\langle P \rho\left(\mathcal{O}^{1}\right), \quad P \rho(1+i)\right\rangle=G
$$

as a subgroup of index 2. But $P \rho(1+i)$ also normalizes $P \rho\left(\Gamma_{1}\right)$ and

$$
\left\langle P \rho\left(\Gamma_{1}\right), P \rho(1+i)\right\rangle \cong S_{4}
$$

from the description above. Thus $S_{4} \subset G$.

Next, in the case where $\mathbf{Q}(\sqrt{5}) \subset k$, if we let

$$
\mathcal{O}=R_{k}\left[1, i, 1 / 2\left(\tau+\tau^{-1} i+j\right), 1 / 2\left(-\tau^{-1}+\tau i+i j\right)\right],
$$

then $\mathcal{O}$ is an order in $A$. Note that the sum of the last two elements above is $\tau^{-1}+1 / 2(1+i+j+i j)$ so that $\Gamma_{3} \subset \mathcal{O}^{1}$. Hence $A_{5} \subset P \rho\left(\mathcal{O}^{1}\right)$ as required.

Case where axes of $\mathbf{f}$ and $h \mathbf{f} h^{-1}$ have one common endpoint. This case is handled by the following result.

Lemma 9.5. Let $G$ be a subgroup of an arithmetic Kleinian group with invariant quaternion algebra $A$ over $k$. If $G$ contains a subgroup isomorphic to the $(3,3,3)$, $(2,4,4)$ or $(2,3,6)$ euclidean triangle group, then

$$
A \cong M(2, \mathbf{Q}(\sqrt{-1})) \text { or } A \cong M(2, \mathbf{Q}(\sqrt{-3})) .
$$

Proof. Since $G$ contains a parabolic, a well known criterion for non-cocompact arithmetic groups implies that $A \cong M(2, \mathbf{Q}(\sqrt{-d}))$ for some square-free integer $d$. Then it follows easily from [35] or [39] that $\mathbf{Q}(\sqrt{-1})$ or $\mathbf{Q}(\sqrt{-3})$ is contained in $k G$ and we obtain (9.6).

Lemmas 9.1, 9.3 and 9.5 yield necessary conditions for the existence of a nonsimple elliptic. We conclude this section with a sufficient condition for the existence of a non-simple elliptic.

Lemma 9.7. Suppose that $f$ and $h$ are Möbius transformations and that $f$ is elliptic of order $n \geq 3$. If

$$
\gamma(f, h) \in(\beta(f), 0)
$$

or if

$$
\gamma(f, h)=\beta(f) \text { and } \beta(h) \neq-4,
$$

then the axes of $f$ and $h f h^{-1}$ intersect in a single point.

Proof. Let $\beta=\beta(f)$ and $\gamma=\gamma(f, h)$. Then

$$
\gamma\left(f, h f h^{-1}\right)=\gamma(\gamma-\beta)
$$

and by Lemma 2.4, 


$$
\begin{aligned}
2 \cosh \left(\delta\left(f, h f h^{-1}\right)\right)^{2} & =\cosh \left(2 \delta\left(f, h f h^{-1}\right)\right)+1 \\
& =\left|\frac{4 \gamma\left(f, h f h^{-1}\right)}{\beta(f) \beta\left(h f h^{-1}\right)}+1\right|+\left|\frac{4 \gamma\left(f, h f h^{-1}\right)}{\beta(f) \beta\left(h f h^{-1}\right)}\right|+1 \\
& =\left|\frac{4 \gamma(\gamma-\beta)+\beta^{2}}{\beta^{2}}\right|+\left|\frac{4 \gamma(\gamma-\beta)}{\beta^{2}}\right| \\
& =2\left(\frac{|\gamma-\beta|+|\gamma|}{|\beta|}\right)^{2}
\end{aligned}
$$

from which we obtain

$$
\cosh \left(\delta\left(f, h f h^{-1}\right)\right)=\frac{|\gamma-\beta|+|\gamma|}{|\beta|} .
$$

If $\gamma \in(\beta, 0)$, then $f$ and $h f h^{-1}$ have disjoint fixed points by $(9.8), \delta\left(f, h f h^{-1}\right)=$ 0 by $(9.9)$ and hence axis $(f)$ and $\operatorname{axis}\left(h f h^{-1}\right)$ intersect in a single point in $\mathbf{H}^{3}$. If $\gamma=\beta$ and if $\beta(h) \neq-4$, then (9.8) and the fact that $h$ is not of order 2 imply that $f$ and $h f h^{-1}$ have a single fixed point in common.

\section{Simple AXES IN EXtremal Groups}

Finally, motivated by the search for lower bounds for the volume of hyperbolic 3 -orbifolds, we apply the criteria of $\S 9$ to determine if the generator $f$ in each group $G_{n, i}$ is simple and hence how to compute its volume contribution. If $f$ is simple, we study its precisely invariant collar. Otherwise $G_{n, i}$ has a spherical triangle subgroup with fixed point the center of a precisely invariant ball or a parabolic subgroup in which case the volume is at least .0845 .

Case $n=3$. From Table 6 in $\S 8$ and Lemma 9.1 we can deduce that for $i=6,7,10$ the groups $G_{3, i}$ do not have subgroups isomorphic to $A_{4}, S_{4}$ or $A_{5}$. In addition, $G_{3,12}$ is Fuchsian. Hence $f$ is simple in these four cases. For the other cases we apply Lemma 9.7 with $\beta(f)=-3$ to show that $f$ is not simple by exhibiting a suitable element $h$ in $G_{n, i}$.

1. $G_{3,1}: \gamma(f, g)=-1$ and $\langle f, g\rangle$ is isomorphic to $S_{4}$.

2. $G_{3,2}: \gamma(f, g)=-.3819 \ldots$ and $\langle f, g\rangle$ is isomorphic to $A_{5}$.

3. $G_{3,3}$ : If $h=g f g$, then $\gamma(f, h)=-2.618 \ldots$.

4. $G_{3,4}$ : If $h=g f g f g f g$, then $\gamma(f, h)=-1$.

5. $G_{3,5}:$ If $h=g f g f g$, then $\gamma(f, h)=-2$.

6. $G_{3,8}$ : If $h=g f g f g f^{-1} g f^{-1} g$, then $\gamma(f, h)=-2$.

7. $G_{3,9}$ : If $h=g f g$, then $\gamma(f, h)=-3$ and $\beta(h)=-3$.

8. $G_{3,11}$ : If $h=g f g f g f g f g$, then $\gamma(f, h)=-1$.

9. $G_{3,13}$ : If $h=k f k^{-1} f k$ where $k=g f g f g$. then $\gamma(f, h)=-1$.

10. $G_{3,14}$ : If $h=g f g f g f g$, then $\gamma(f, h)=-2$.

Case $n=4$. The methods here are the same as for $n=3$. In particular, the cases where $i=2,5,6$ are dealt with by Lemma 9.1 as above while $G_{4,10}$ is Fuchsian. Hence $f$ is simple in these four cases. Otherwise Lemma 9.7 with $\beta(f)=-2$ shows that $f$ is not simple when $i \neq 7,11$.

1. $G_{4,1}: \gamma(f, g)=-1$ and $\langle f, g\rangle$ is isomorphic to $S_{4}$.

2. $G_{4,3}$ : If $h=g f g f g$, then $\gamma(f, h)=-1$.

3. $G_{4,4}$ : If $h=g f g$, then $\gamma(f, h)=-2$ and $\beta(h)=-2$.

4. $G_{4,8}$ : If $h=g f g f g f g$, then $\gamma(f, h)=-1$. 
5. $G_{4,9}$ : If $h=g f g f g$, then $\gamma(f, h)=-2$ and $\beta(h)=2 i$.

6. $G_{4,12}$ : If $h=g f g f g f^{-1} g f^{-1} g$, then $\gamma(f, h)=-1$.

7. $G_{4,13}$ : If $h=g f g f g f g f g$, then $\gamma(f, h)=-1$.

Case $n=5$. In this case, the only group which can be dealt with directly by the methods of $\S 9$ is $G_{5,7}$. In this example, although there is only dyadic ramification, the algebra is not isomorphic to

$$
\left(\frac{-1,-1}{k_{5,7}}\right)
$$

as is shown by Lemma 9.3 .

Finally since $G_{5,5}$ is Fuchsian we conclude that $f$ is simple when $i=4,5,7$. We can then apply Lemma 9.7 with $\beta(f)=-1.3819 \ldots$ to show that $f$ is not simple when $i \neq 8,9,11$.

1. $G_{5,1}: \gamma(f, g)=-.38196 \ldots$ and $\langle f, g\rangle$ is isomorphic to $A_{5}$.

2. $G_{5,2}$ : If $h=g f g$, then $\gamma(f, h)=-1$.

3. $G_{5,3}$ : If $h=g f g f g$, then $\gamma(f, h)=-.38196 \ldots$

4. $G_{5,6}$ : If $h=g f g f g$, then $\gamma(f, h)=-1$.

5. $G_{5,10}$ : If $h=g f g f g f^{-1} g f^{-1} g$, then $\gamma(f, h)=-.38196 \ldots$

6. $G_{5,12}$ : If $h=g f g f g f g$, then $\gamma(f, h)=-1$.

Case $n=6$. When $n=6$, Lemma 9.5 implies that the only cases for which $f$ cannot be simple are $G_{6,1}$ and $G_{6,3}$. The calculations for these using Lemma 9.7 with $\beta(f)=-1$ are given below.

1. $G_{6,1}$ : If $h=g f g$, then $\gamma(f, h)=-1$ and $\beta(h)=-1$.

2. $G_{6,3}$ : If $h=g f g f g$, then $\gamma(f, h)=-1$ and $\beta(h)=.5+i 2.598 \ldots$

The six groups $G_{4,7}, G_{4,11}, G_{5,4}, G_{5,8}, G_{5,9}$ and $G_{5,11}$ have not been treated in the above calculations. The program of Jones and Reid mentioned in $\S 8$ shows that $f$ is simple in all of these groups.

We summarize these results in Table 12 .

TABLE 12. $f$ simple elliptic

\begin{tabular}{|c|c|c|c|c|}
\hline$i$ & $G_{3, i}$ & $G_{4, i}$ & $G_{5, i}$ & $G_{6, i}$ \\
\hline 1 & $S_{4}$ & $S_{4}$ & $A_{5}$ & No \\
\hline 2 & $A_{4}$ & Yes & No & Yes \\
\hline 3 & No & No & No & No \\
\hline 4 & No & No & Yes & Yes \\
\hline 5 & No & Yes & Fuch. & Yes \\
\hline 6 & Yes & Yes & No & Yes \\
\hline 7 & Yes & Yes & Yes & Fuch. \\
\hline 8 & No & No & Yes & Yes \\
\hline 9 & No & No & Yes & -- \\
\hline 10 & Yes & Fuch. & No & -- \\
\hline 11 & No & Yes & Yes & -- \\
\hline 12 & Fuch. & No & No & -- \\
\hline 13 & No & No & -- & -- \\
\hline 14 & No & -- & -- & -- \\
\hline
\end{tabular}




\section{Commutator Parameter Diagrams}

We conclude this paper with four diagrams which result from the disk covering argument described in $\S 2$ for the cases where $n=3,4,5,6$. They represent the only possible values for the commutator parameter $\gamma=\gamma(f, g)$ of a discrete group $\langle f, g\rangle$ for which

1. $f$ is an elliptic of order $n$,

2. $\gamma$ lies in the union of the indicated disks.

The values in these diagrams which correspond to subgroups of arithmetic groups are listed in Tables 1, 2, 3 and 4 in $\S 6$. As noted earlier in $\S 2$, all of the values in the $n=3$ diagram have this property.

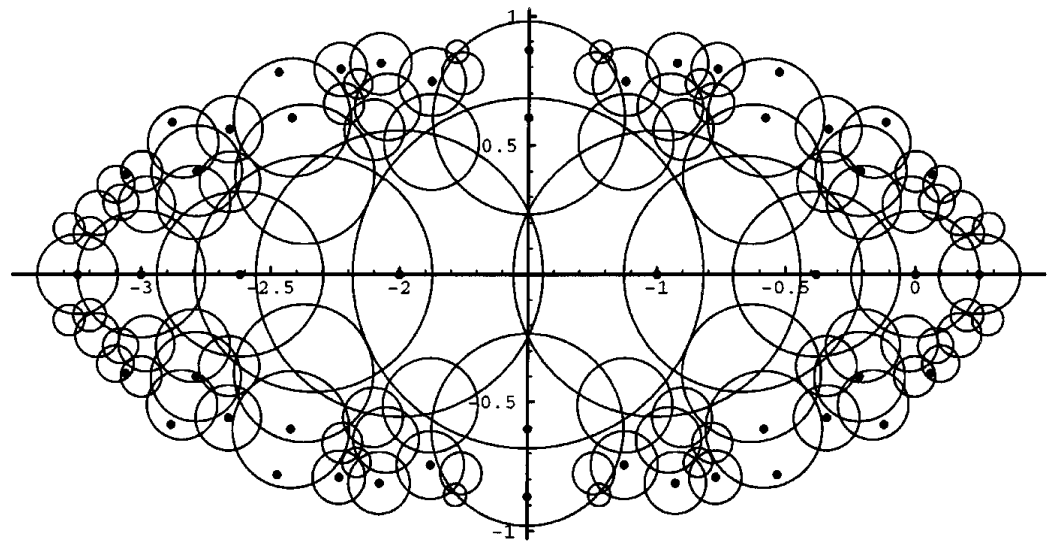

Figure 2. Only possible values for commutator parameter when $n=3$

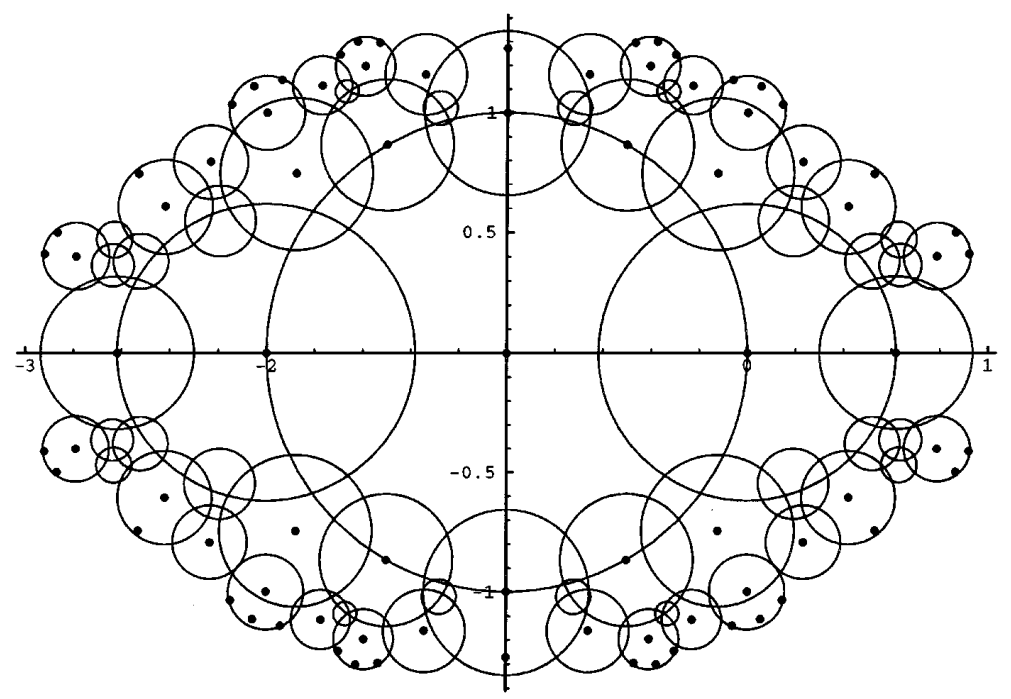

Figure 3. Only possible values for commutator parameter when $n=4$ 


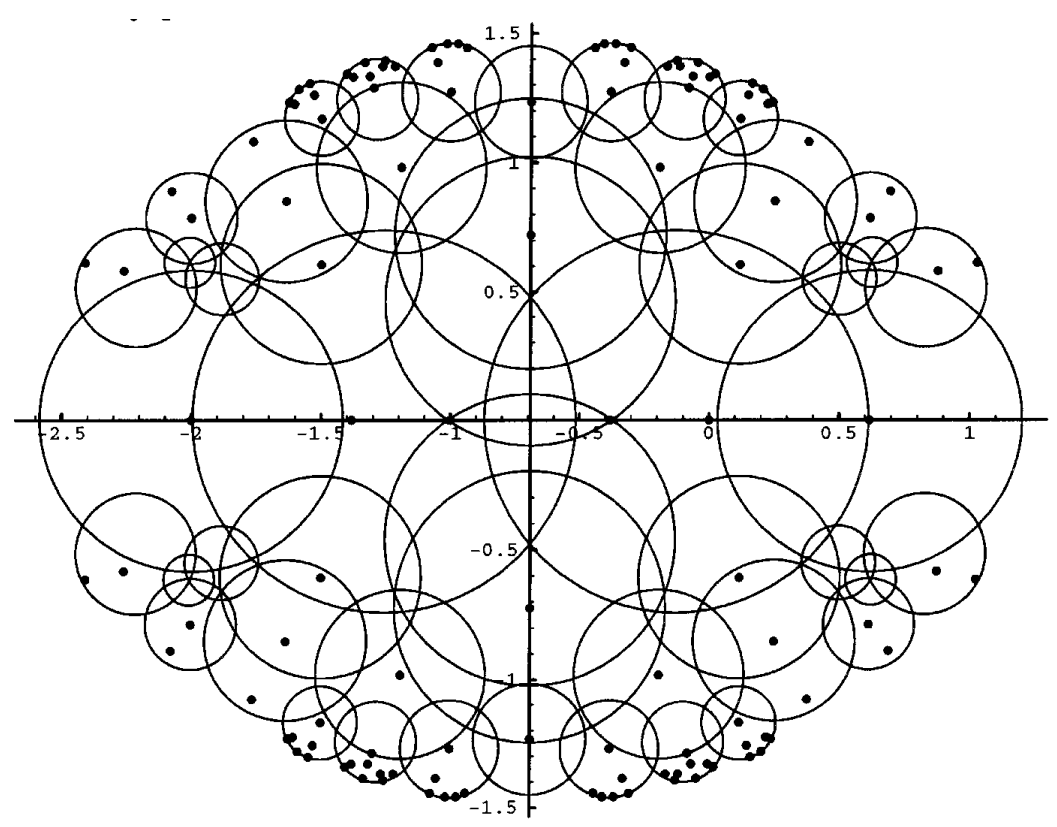

Figure 4. Only possible values for commutator parameter when $n=5$

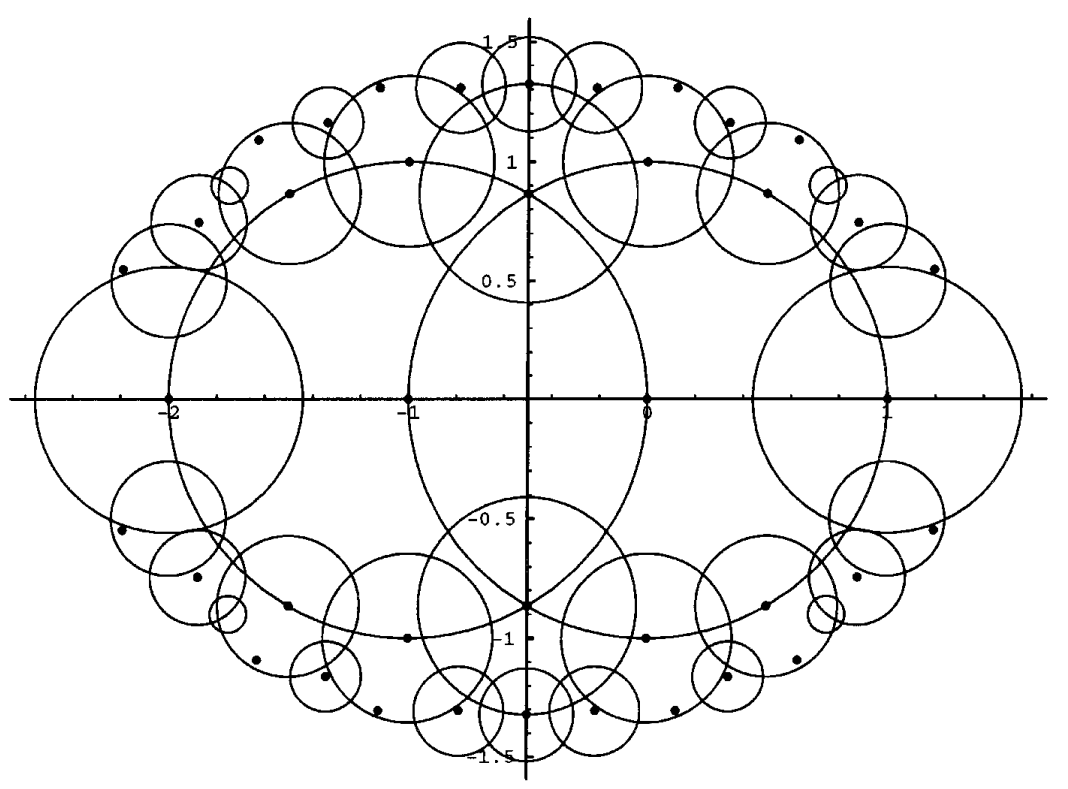

Figure 5. Only possible values for commutator parameter when $n=6$ 


\section{REFERENCES}

1. C. Adams, The noncompact hyperbolic 3-manifold of minimal volume, Proc. Amer. Math. Soc. 100 (1987) 601-606. MR 88m:57018

2. C. Adams, Limit volumes of hyperbolic 3-orbifolds, J. Diff. Geom. 34 (1991) 115-141. MR 92d:57029

3. H. Bass, Groups of integral representation type, Pacific J. Math. 86 (1980) 15-51. MR 82c:20014

4. A. F. Beardon, The geometry of discrete groups, Springer-Verlag 1983. MR 85d:22026

5. A. Borel, Commensurability classes and volumes of hyperbolic 3-manifolds, Ann. Scuola Norm. Sup. Pisa 8 (1981) 1-33. MR 82j:22008

6. L. A. Best, On torsion-free discrete subgroups of $P S L_{2}(\mathbf{C})$ with compact orbit space, Can. J. Math. 23 (1971) 451-460. MR 44:1767

7. C. Cao, On three-generator Möbius groups, New Zealand Math. J. 23 (1994) 111-120. MR 96b:30093

8. T. Chinburg A small arithmetic hyperbolic three-manifold, Proc. Amer. Math. Soc. 100 (1987) 140-144. MR 88b:57043

9. T. Chinburg and E. Friedman, The smallest arithmetic hyperbolic 3-orbifold, Invent. Math. 86 (1986) 507-527. MR 88a:22022

10. T. Chinburg, E. Friedman, K. N. Jones and A. W. Reid, The smallest volume arithmetic hyperbolic 3-manifold, Preprint.

11. M. Culler and P. Shalen, Paradoxical decompositions, 2-generator Kleinian groups, and volumes of hyperbolic 3-manifolds, J. Amer. Math. Soc. 5 (1992) 231-288. MR 93a:57017

12. D. A. Derevnin and A. D. Mednykh, Geometric properties of discrete groups acting with fixed points in Lobachevsky space, Dokl. Akad. Nauk SSSR 300 (1988) 614-617. MR 90a:30131

13. D. J. H. Garling, A course in Galois theory, Camb. Univ. Press 1986. MR 88d:12007

14. D. Gabai, On the geometric and topological rigidity of hyperbolic 3-manifolds, J. Amer. Math. Soc. 10 (1997), 37-74. CMP 97:02

15. D. Gabai, R. Meyerhoff and N. Thurston, Personal Communication.

16. F. W. Gehring and G. J. Martin, Axial distances in discrete Möbius groups, Proc. Natl. Acad. Sci. USA 89 (1992) 1999-2001. MR 92m:30082

17. F. W. Gehring and G. J. Martin, 6-torsion and hyperbolic volume, Proc. Amer. Math. Soc. 117 (1993) 727-735. MR 93d:30056

18. F. W. Gehring and G. J. Martin, Commutators, collars and the geometry of Möbius groups, J. d'Analyse Math. 63 (1994) 175-219. MR 96c:30040

19. F. W. Gehring and G. J. Martin, On the minimal volume hyperbolic 3-orbifold, Math. Res. Letters 1 (1994) 107-114. MR 95b:30072

20. F. W. Gehring and G. J. Martin, On the Margulis constant for Kleinian groups, I. MSRI preprint \# 038-95

21. F. W. Gehring and G. J. Martin, Commutator spectra for discrete groups with an elliptic generator (in preparation).

22. F. W. Gehring and G. J. Martin, Tetrahedral, octahedral and icosahedral subgroups of a Kleinian group (in preparation).

23. F. W. Gehring and G. J. Martin, The volume of hyperbolic 3-folds with p-torsion, $p \geq 6$. Centre for Mathematics and its Applications, Preprint (1995).

24. F. W. Gehring and G. J. Martin, Precisely invariant collars and the volumes of hyperbolic 3-folds. Centre for Mathematics and its Applications, Preprint (1995).

25. F. W. Gehring and G. J. Martin, Torsion and volume in hyperbolic 3-folds (to appear).

26. H. J. Godwin, On quartic fields with signature one and small discriminant, Quart. J. Math. Oxford (2) 8 (1957) 214-222. MR 20:3844

27. H. M. Hilden, M-T. Lozano and J. M. Montesinos, A characterization of arithmetic subgroups of $\mathrm{SL}(2, \boldsymbol{R})$ and $\mathrm{SL}(2, \boldsymbol{C})$, Math. Nach. 159 (1992) 245-270. MR 94i:20088

28. R. Horowitz, Characters of free groups represented in the two-dimensional special linear group, Comm. Pure Appl. Math. 25 (1972) 635-649. MR 47:3542

29. K. N. Jones and A. W. Reid, Non-simple geodesics in hyperbolic 3-manifolds, Math. Proc. Camb. Phil. Soc. 116 (1994), 339-351. MR 95e:57025

30. C. Maclachlan and A. W. Reid, Commensurability classes of arithmetic Kleinian groups and their Fuchsian subgroups, Math. Proc. Camb. Phil. Soc 102 (1987) 251-257. MR 88j:20040 
31. C. Maclachlan and A.W. Reid, The arithmetic structure of tetrahedral groups of hyperbolic isometries, Mathematika 36 (1989) 221-240. MR 91b:11055

32. A. Marden, The geometry of finitely generated Kleinian groups, Annals of Math. 99 (1974) 383-462. MR 50:2485

33. B. Maskit, Kleinian groups, Springer-Verlag 1988. MR 90a:30132

34. R. Meyerhoff, The cusped hyperbolic 3-orbifold of minimum volume, Bull. Amer. Math. Soc. 13 (1985) 154-156. MR 87b:22022

35. W. D. Neumann and A. W. Reid, Arithmetic of hyperbolic 3-manifolds. In TOPOLOGY '90, Proceedings of the Research Semester in Low-Dimensional Topology at Ohio State University, 273-310. Editors, B. Apanasov, W. D. Neumann, A. W. Reid and L. Siebenmann, De Gruyter Verlag 1992. MR 94c:57024

36. M. Pohst, P. Weiler and H. Zassenhaus On effective computation of fundamental units II, Math. Comp. 38 (1982), 293-329. MR 83e:12005b

37. A. W. Reid, Ph. D Thesis, University of Aberdeen, 1987.

38. A. W. Reid, A note on trace-fields of Kleinian groups, Bull. London Math. Soc. 22 (1990) 349-352. MR 91d:20056

39. A. W. Reid, Arithmeticity of Knot Complements, J. London Math. Soc.(2) 43 (1991) 171-184. MR 92a:57011

40. K. Takeuchi, A characterization of arithmetic Fuchsian groups, J. Math. Soc. Japan 27 (1975) 600-612. MR 53:2842

41. W.P. Thurston The geometry and topology of 3-manifolds Princeton Lecture Notes, 1977.

42. M-F. Vignéras, Arithmétique des algèbres de quaternions. Lecture Notes in Math., vol. 800, Springer-Verlag 1980. MR 82i: 12016

43. E. B. Vinberg, Rings of definition of dense subgroups of semisimple linear groups, Math. USSR Izvestija 35 (1971) 45-55. MR 43:4929

Department of Mathematics, University of Michigan, Ann Arbor, Michigan 481091003

E-mail address: fgehring@math.1sa.umich.edu

Department of Mathematics, University of Aberdeen, Aberdeen, Scotland

E-mail address: cmac@math.aberdeen.ac.uk

Department of Mathematics, University of Auckland, Auckland, New Zealand and Australian National University, Canberra, Australia

E-mail address: martin@math.auckland.ac.nz

Department of Mathematics, University of Texas, Austin, Texas 78712

E-mail address: areid@math.utexas.edu 